\title{
ScienceImmunology
}

Submitted Manuscript: Confidential

template updated: July 52016

\section{Title: $S$. Typhi Vi capsule prime-boost vaccination induces convergent and functional antibody responses}

Authors: Lindsay C. Dahora ${ }^{1,2} \dagger^{*}$, Marije K. Verheul ${ }^{11} \uparrow$, Katherine L. Williams ${ }^{12}$, Celina $\operatorname{Jin}^{11}$, Lisa Stockdale $^{11}$, Guy Cavet ${ }^{12}$, Eldar Giladi ${ }^{12}$, Jennifer Hill ${ }^{11}$, Dongkyoon Kim ${ }^{12}$, Yvonne Leung $^{12}$, Benjamin G. Bobay ${ }^{7,8,10}$, Leonard D. Spicer ${ }^{7,8,10}$, Sheetal Sawant ${ }^{1,4}$, Sjoerd Rijpkema ${ }^{13}$, S. Moses Dennison ${ }^{1,4}$, S. Munir Alam ${ }^{3,5,9}$, Andrew J. Pollard ${ }^{11 \Phi}$, Georgia D. Tomaras 1, 2, 4, 6* $\Phi$

\section{Affiliations:}

${ }^{1}$ Center for Human Systems Immunology ${ }^{2}$ Departments of Immunology, ${ }^{3}$ Medicine, ${ }^{4}$ Surgery, ${ }^{5}$ Pathology, ${ }^{6}$ Molecular Genetics and Microbiology, ${ }^{7}$ Biochemistry, ${ }^{8}$ Radiology, Duke University, Durham, NC, United States

${ }^{9}$ Duke Human Vaccine Institute, Duke University, Durham, NC, United States

${ }^{10}$ Duke University NMR Center, Duke University Medical Center, Durham, NC, United States

${ }^{11}$ The Oxford Vaccine Group, Department of Pediatrics, University of Oxford, and the NIHR Oxford Biomedical Research Center, Oxford, United Kingdom

${ }^{12}$ Atreca, Inc., San Carlos, CA, United States

${ }^{13}$ Division of Bacteriology, National Institute of Biological Standards and Control, Potters Bar, United Kingdom

*Corresponding authors: Emails: 1cd26@duke.edu,gdt@duke.edu

$\dagger$ Authors contributed equally

$\Phi$ Authors contributed equally.

One Sentence Summary: Prime-boost vaccination with $S$. Typhi Vi capsule induces convergent antibodies whose function depends on specificity and avidity.

Abstract:

Vaccine development to prevent Salmonella Typhi infections has accelerated over the past decade, resulting in licensure of new vaccines, which use the Vi polysaccharide (Vi PS) of the bacterium conjugated to an unrelated carrier protein, as the active component. Antibodies elicited by these vaccines are important for mediating protection against typhoid fever. However, the characteristics of protective and functional $\mathrm{Vi}$ antibodies are unknown. In this study, we investigated the human antibody repertoire, avidity maturation, epitope specificity, and function after immunizations with a single dose of Vi-tetanus toxoid conjugate vaccine (Vi-TT), and after a booster with plain Vi PS (Vi-PS). The Vi-TT prime induced an IgG1-dominant response, while the Vi-TT prime followed by the Vi-PS boost induced $\mathrm{IgG} 1$ and $\mathrm{IgG} 2$ antibody production. B-cells from recipients who received both prime and boost showed evidence of convergence with shared $\mathrm{V}$ gene usage and CDR3 characteristics. The detected $\mathrm{Vi}$ antibodies showed heterogeneous avidity ranging from 
$10 \mu \mathrm{M}-500 \mathrm{pM}$, with no evidence of affinity maturation after the boost. Vi-specific antibodies mediated Fc effector functions, which correlated with antibody dissociation kinetics but not with association kinetics. We identified antibodies induced by prime and boost vaccines that recognized subdominant epitopes, indicated by binding to the de- $O$-acetylated Vi backbone. These antibodies also mediated Fc-dependent functions, such as complement deposition and monocyte phagocytosis. Defining strategies on how to broaden epitope targeting for Salmonella Typhi Vi and enriching for antibody $\mathrm{Fc}$ functions that protect against typhoid fever will advance the design of high efficacy Vi vaccines for protection across diverse populations.

\section{Introduction}

Vaccines are designed to induce a protective immune response against pathogens through the induction of functional antibodies (1). Correlates or surrogates of protection are often based on antibody levels (e.g. Haemophilus influenzae type b, hepatitis B vaccines) (2), though in many cases, protective antibody thresholds are difficult to reproduce. Few vaccines have mechanistic correlates, such as those measured in a serum bactericidal assay (e.g. meningococcal vaccines) or opsonophagocytosis assay (e.g. pneumococcal vaccines) $(1,3)$. As such, increased attention is needed on not only the quantity but also the quality of the antibody response. Where quantitative antibody features fail, qualitative features such as specificity and affinity play a significant role in protection against various pathogens including HIV-1, Malaria, Zika, and others (4-7). In addition, antibody repertoire sequencing and single cell B-cell receptor (BCR) sequencing have provided insight into antibody development and selection upon immunization. Studying antibody responses after immunization and infection is therefore important to determine the antibody specificities that impart protection or control, and ultimately for the development and optimization of vaccines.

One of the more recently developed vaccines for the prevention of typhoid fever is a Vi typhoid conjugate vaccine (Vi-TCV), in which Vi capsular polysaccharide antigen (Vi PS) is conjugated to tetanus toxoid (Vi-TT). Vi-TT induces immune responses that target Vi PS of Salmonella enterica subspecies enterica serovar Typhi (S. Typhi) (8). This bacterial pathogen infects 10-13 
million people annually (9) and is evolving to display extensive antibiotic resistance $(10,11)$. Immunogenicity of Vi-TT was established in a non-endemic population using a controlled human infection model (CHIM), where the efficacy was found to be comparable to the licensed plain Vi PS vaccine (Vi-PS) (12). Following this, a Vi-TT efficacy of $82 \%$ was observed in a randomized, controlled trial in typhoid-endemic Nepal (13). Both studies reported an increase in anti-Vi IgG antibodies after immunization, however, estimates on thresholds of $\mathrm{Vi} \operatorname{IgG}$ required for protection have been difficult to reproduce for decades $(14,15)$. Recently, we reported that Vi PS specific IgG1 avidity and IgA magnitude and fold-change were associated with protection in a CHIM study $(16,17)$. Although Vi vaccination is widely used to protect against typhoid fever, data on repertoire and functionality of antibodies induced after Vi immunization are currently limited. Understanding how to broaden the specificities and functional antibody repertoire will inform further immunogen design to protect against typhoid fever.

The Vi PS antigen constitutes one of the major virulence factors of $S$. Typhi as it shields the bacterial cell from the innate immune system upon entry through the gastrointestinal tract $(18,19)$. The Vi PS antigen is a linear homopolymer of $\alpha-1 \rightarrow 4-$ galacturonic acid with variable $O$ acetylation (60-90\% in naturally occurring $S$. Typhi) at the C-3 position and a fixed $\mathrm{N}$-acetyl at the C-2 position $(20,21)$. Immunogenicity and antigenicity data have shown that the immunodominant epitope of Vi PS is the C-3 $O$-acetyl group. This has been explained through molecular dynamics simulations showing that the $O$-acetyl is solvent exposed and bulky, thus burying the polysaccharide backbone and occluding alternative epitopes (22). However, partial de- $O$ acetylation ( $45 \% O$-acetylated $\mathrm{Vi}$ ) has been shown to increase immunogenicity of Vi PS antigen (20) possibly owing to increased flexibility in the polysaccharide backbone (22). These studies have resulted in a WHO standard for formulation of Vi vaccines to include Vi PS antigen with at 
least $52 \%(2 \mathrm{mmol} / \mathrm{g} \mathrm{Vi})$ of monomers $O$-acetylated at C-3 (23). Despite the immunodominance of the C-3 $O$-acetyl group, studies have shown that the vaccine still induces rabbit and human sera that exhibit binding to de- $O$-acetylated Vi PS antigen, albeit at very low levels $(20,22)$. The binding to de- $O$-acetylated Vi PS antigen following vaccination indicates that vaccine-elicited antibodies also target non- $O$-acetyl alternative epitopes, likely the $\mathrm{C}-2 \mathrm{~N}$-acetyl and/or the C-5 carboxyl, which has been shown by one mouse monoclonal antibody $(24,25)$. These epitopes may be subdominant but still solvent exposed (SEE) or buried beneath the bulky $O$-acetyl group and occluded (SEO). To date, the repertoire of antibodies generated toward Vi PS antigen and their functional relevance in humans has not been explored.

Here we evaluate the B-cell repertoire of antibodies elicited by $\mathrm{Vi}$ vaccination in humans, determine which epitope specificities are present, investigate their avidity for Vi PS antigen, and establish their ability to mediate Fc-dependent effector functions. We show evidence of a heterogeneous antibody response with regards to antibody repertoire, IgG subclass distribution, and affinity maturation following both prime (Vi-TT) and boost (Vi-PS). We identified Vi PS antigen-specific antibodies using selection strategies based on sequence convergence and the expansion and persistence of B-cell lineages. At least four subdominant, alternative epitopes targeted by vaccine-elicited antibodies were identified including two epitopes that are shielded by the $O$-acetyl group and two epitopes that are exposed regardless of the presence of $O$-acetyl. Antibodies targeting the exposed epitopes can mediate antibody-dependent monocyte phagocytosis (ADMP) and complement deposition (ADCD) with a magnitude that significantly correlates with antibody-antigen off-rate kinetics.

\section{Results}



heavy chain and subclass usage

114 A total of twelve participants were recruited to be immunized with Vi-TT, followed by a Vi-PS

115 boost, and ten participants completed the study. A subset of eight participants was selected based on peripheral blood mononuclear cell (PBMC) availability. Paired data after both immunizations

117 was available for only three of these eight participants, while for the remaining five participants, 118 data was available only after the Vi-PS boost. IgG-positive plasmablasts collected from 119 participants 7 days post-prime with Vi-TT $(\mathrm{n}=3)$ and 7 days post-boost with Vi-PS $(\mathrm{n}=8)$ were used for single-cell BCR sequencing (fig. S1). Importantly, humoral responses measured following Vi-PS boost represent both memory B-cell responses induced by the Vi-TT prime in addition to naïve B-cell responses induced by the Vi-PS boost. As Ig subclasses are major determinants of antibody functionality, we investigated the subclass distribution within identified BCRs. After ViTT primary immunization, the predominant subclass was IgG1 $(81.9 \%-86.5 \%)$, with a small percentage of IgG2 (10.2\% - 15.9\%). In the paired samples after the Vi-PS boost, IgG1 (50.1\%$65.6 \%)$ remained the most prevalent, but increased percentages of IgG2 antibodies $(28.1 \%$ $46.5 \%$ ) were observed. IgG2 sequences were also prevalent in the additional non-paired Vi-PS samples $(38.8 \%$ - 80.0\%), compared with $\operatorname{IgG} 1$ (17.2\% - 49.3\%). IgG3 and IgG4 formed a very small proportion of the total BCRs identified (up to 13.2\%) (Fig. 1A). 
A.

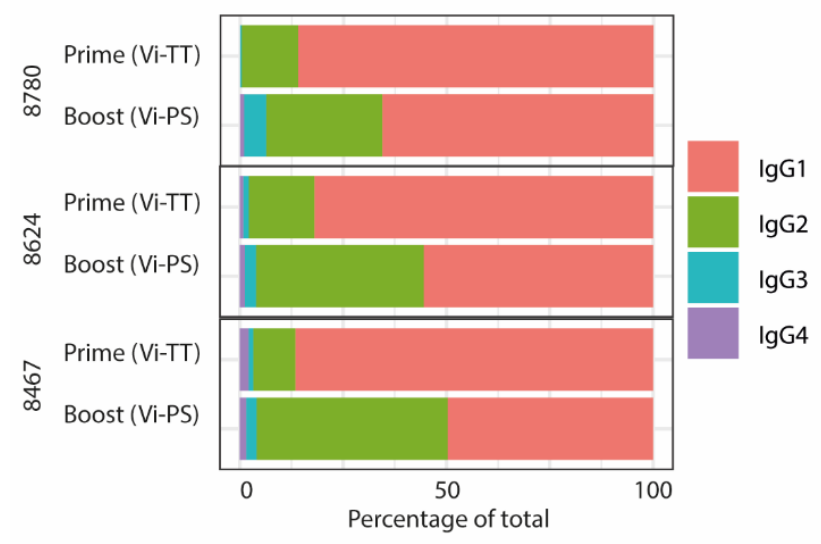

c.

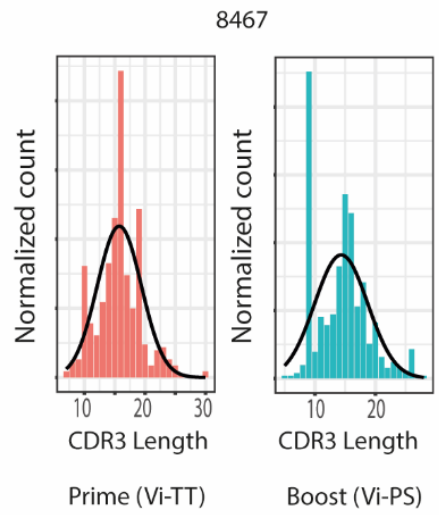

D.

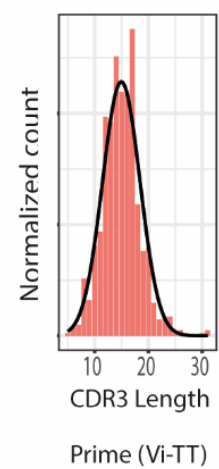

B.

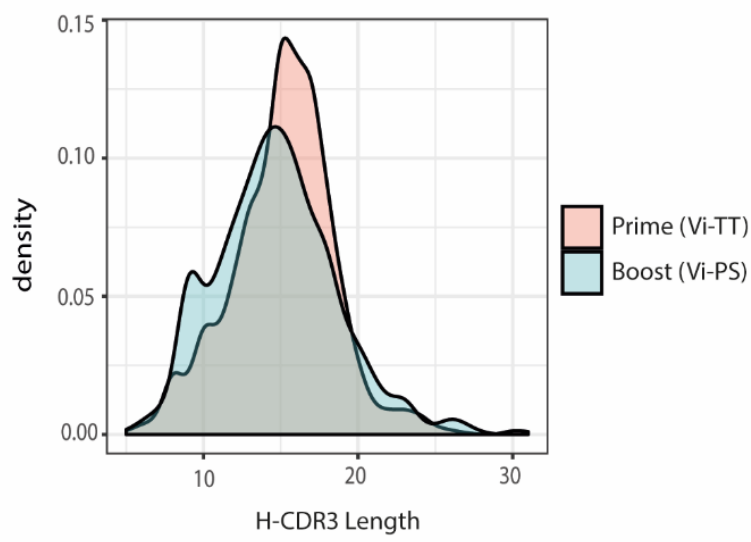

8624

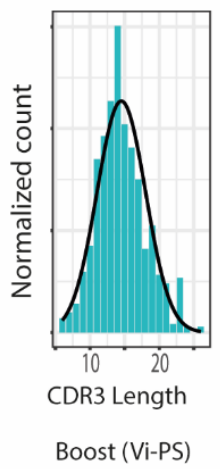

E.

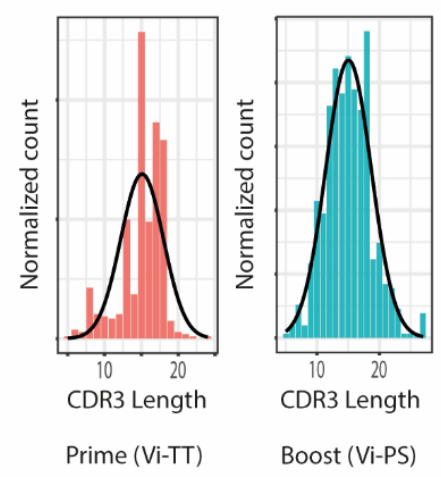

F.
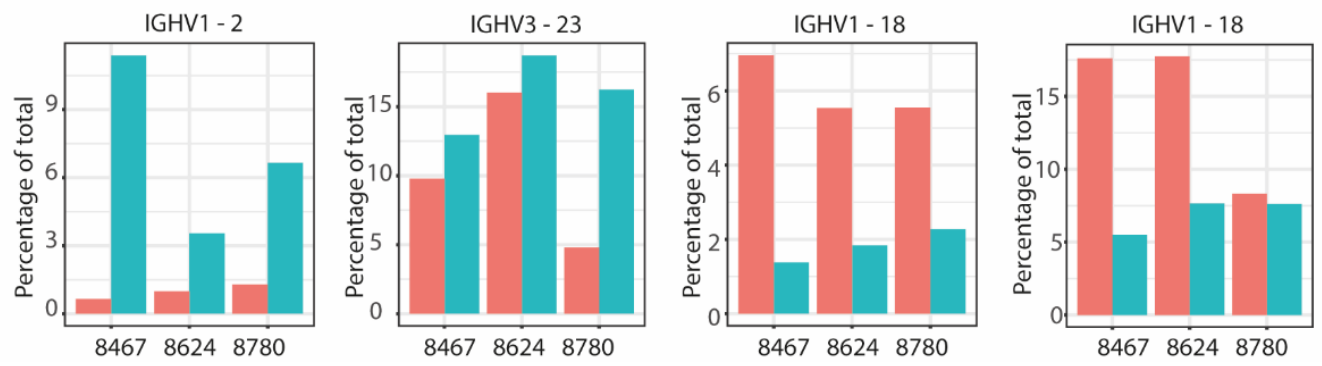

Prime (Vi-TT)

Boost (Vi-PS)

Fig. 1. Sequence characteristics of BCRs from IgG-positive plasmablasts isolated after Vi-

TT primary or Vi-PS boost immunization A) Subclass distribution for $n=3$ participants, in response to prime (Vi-TT) and boost (Vi-PS) in a paired fashion. B) Heavy chain CDR3 length after Vi-TT prime $(n=3)$ or Vi-PS-boost $(n=3)$, showing paired samples only. C) - E) Individual

140 heavy chain CDR3 length after Vi-TT prime or Vi-PS boost. Grey lines are based on a normal

141 distribution with the mean and standard deviation of the CDR3 length data for that particular

142 sample. F) Ig Heavy chain variable region usage in paired samples, comparing Vi-TT prime and 
Vi-PS boost. Four heavy chain regions of interest are shown. Further regions are shown in supplementary figure S4.

To further characterize the observed heavy chain sequences, additional features corresponding to functionally mature antibodies were investigated. The complementarity-determining region 3 (CDR3) lengths after both Vi-TT prime and Vi-PS boost immunizations showed the expected Gaussian distribution, with little difference (Fig. 1B). The heavy chain CDR3 length distributions for individual vaccinees included sharp peaks at particular lengths, suggesting that there were marked expansions of specific B-cell clones after immunization (Fig. 1C-E, fig. S2). All six CDR3 length distributions deviate from a normal distribution (Shapiro-Wilk Normality test $(\mathrm{p}<0.001$ for each)). In addition, some of the heavy chain $\mathrm{V}$ genes were over-represented after either the Vi-TT prime or the Vi-PS boost immunization (Fig. 1F, fig. S3), suggesting that the prime and booster shots elicit antibody responses with different characteristics. These differences (Fig. 1) could be due in part to recall of memory cells following the boost. Thus, the Vi-TT primary immunization largely induced an IgG1 immune response, while the Vi-PS boost induced a response with both $\mathrm{IgG} 1$ and $\mathrm{IgG} 2$. In addition, the sequence analysis indicated that clonal expansion occurred after both immunizations.

Selection based on convergence, expansion and persistence results in successful identification of Vi-specific antibodies

To identify Vi PS antigen-specific antibodies within the studied repertoires, antibodies were selected for expression as recombinant protein based on three approaches. Antibodies from 
166 individual participants were grouped into families based on the same predicted $\mathrm{V}_{\mathrm{H}} / \mathrm{J}_{\mathrm{H}}$ and $\mathrm{V}_{\mathrm{L}} / \mathrm{J}_{\mathrm{L}}$

167 germline gene usage and with $\mathrm{V}_{\mathrm{H}}$ and $\mathrm{V}_{\mathrm{L}}$ CDR3 sequences of equal lengths. The first approach

168 was to favor the largest expanded families. As expansion of antigen-specific plasmablasts is

169 expected seven days after immunization, larger families are more likely to represent the humoral

170 response to the vaccine. Most families contained only one or two sequences, with only a small

171 proportion showing greater expansion (Fig. 2A, fig. S4). Only families expanded following the

172 Vi-PS booster immunization were selected in this approach. The second approach was to identify

173 antibody family persistence by selecting families which were observed after the prime

174 immunization and also present and expanded in the same participant after the Vi-PS boost (Fig.

175 2B). The persistent observation of a family after both immunizations is consistent with activation

176 by the Vi component of both vaccines instead of the TT component of the Vi-TT prime. In the

177 third approach, antibodies were prioritized based on convergence across participants (Fig. 2C). A

178 total of 96 families were selected based on a combination of expansion, persistence, and 179 convergence (Fig. 2D), and one representative of each family was picked to generate 96 180 monoclonal antibodies (mAbs). 
A.

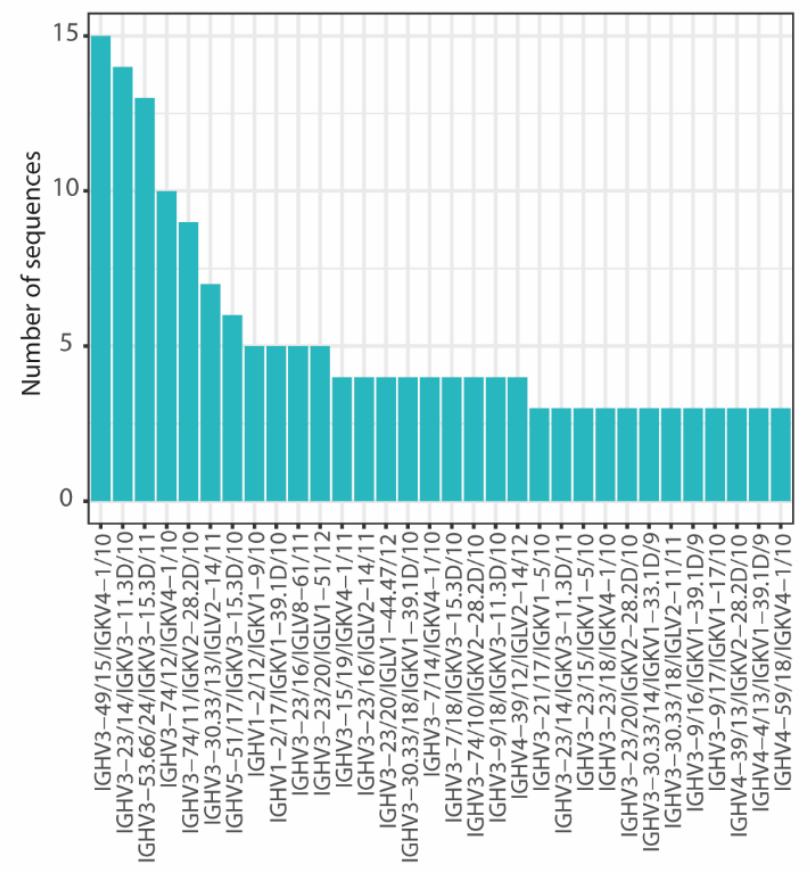

Family

D.

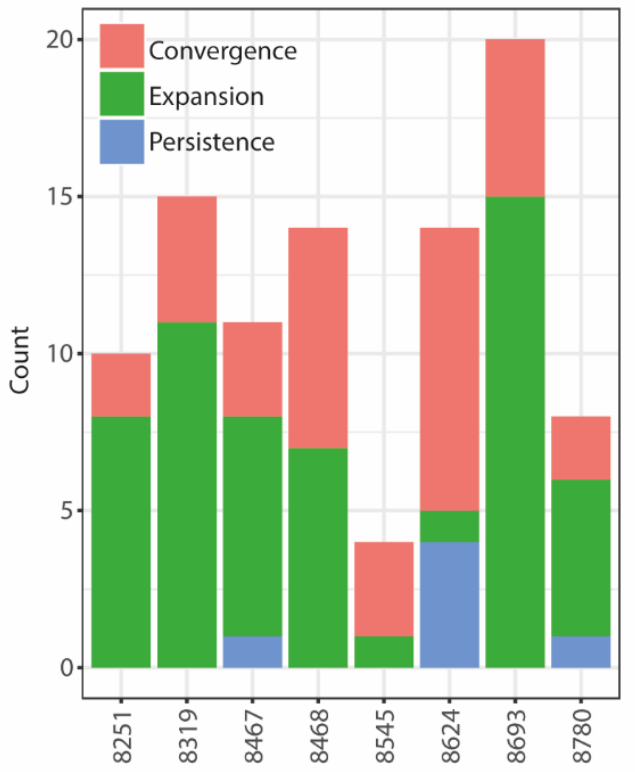

F.

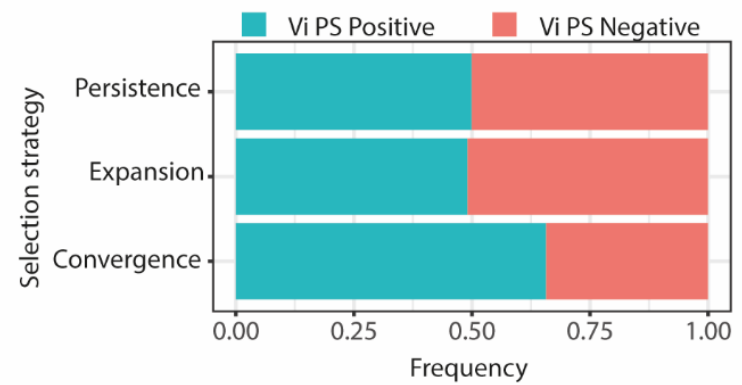

B.

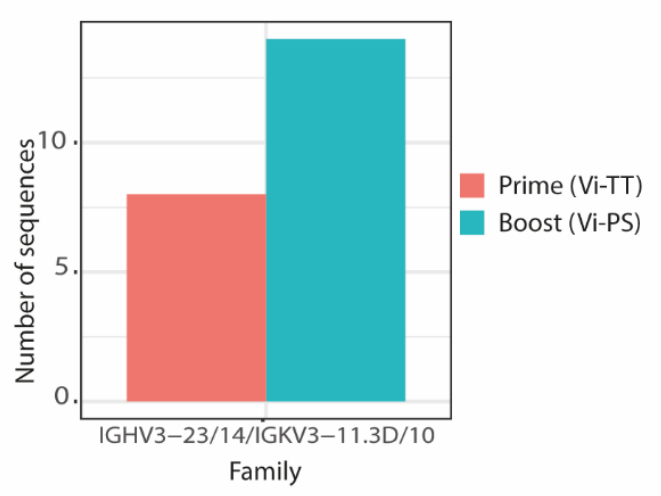

C.

ID Family CDR3

8468 IGHV3-23/14/IGKV3-11.3D/10 ARAKTGTIRAFDIW 8624 IGHV3-23/14/IGKV3-11.3D/10 ARGQTGTIRNFDYW

E.

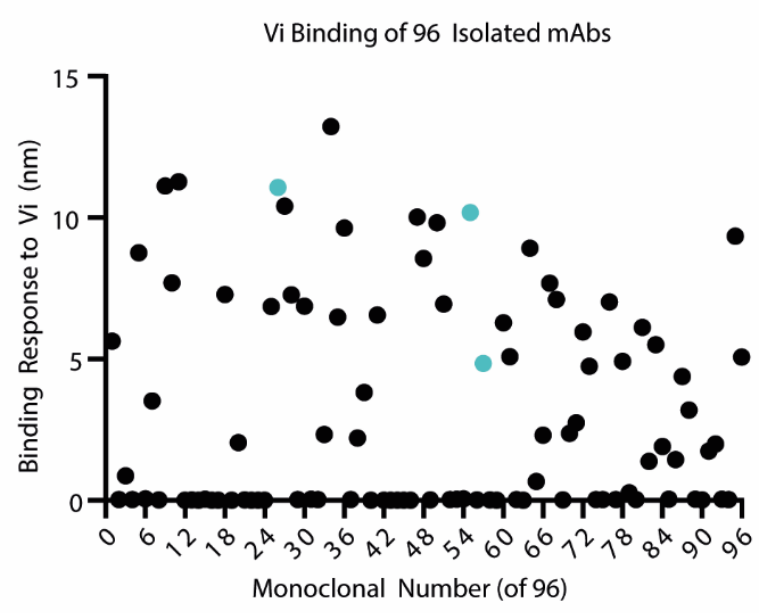

G.

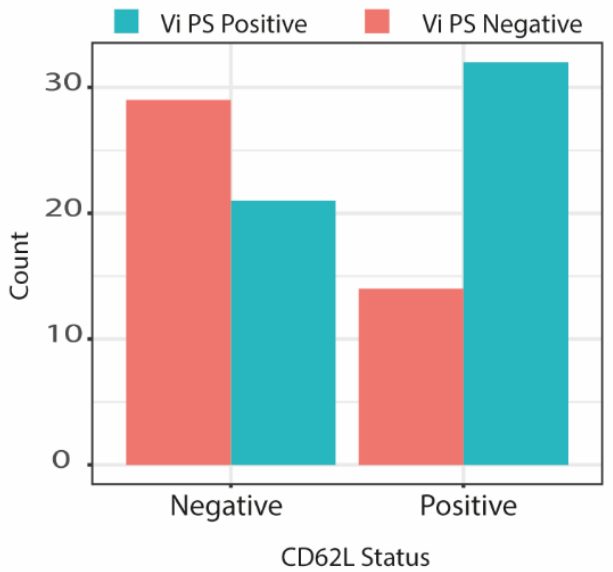


Fig. 2. Selection strategy for the identification of Vi-specific monoclonal antibodies A) Family size of sequences identified in participant 8624 after Vi-PS boost. Families with fewer than 3 sequences were excluded for the purpose of visualization. B) Example of a family from 8624, selected based on a combination of expansion and persistence. C). Example of a selected convergent antibody pair. D) Indicates the strategy used to select each of the 96 monoclonal antibodies, split by each of the different donors. Antibodies categorized on the basis of persistence were also expanded. E) Vi binding of $n=96$ isolated monoclonal antibodies by BLI where blue dots indicate mAbs isolated from prime time-point. F) Frequency of Vi binding monoclonal antibodies for each of the selection strategies employed, as measured by BLI. G) Shows Vi Status of the $n=96$ selected monoclonals, stratified by CD62L status.

Out of 96 generated mAbs, 53/96 (55\%) were positive for Vi PS antigen binding by BioLayer Interferometry (BLI) ranging from low level binding at the $0.08 \mathrm{~nm}$ positivity cut-off to high binding with a maximum of $13.22 \mathrm{~nm}$ (Fig. 2E). For one out of eight participants, none of the selected antibodies bound to Vi PS antigen. Antibodies selected for convergence had the highest rate of Vi PS antigen binding (63\%), followed by $50 \%$ for persistence and $47 \%$ for expansion (Fig. $2 \mathrm{~F}$ ). This is consistent with the induction of convergent antibody responses (similar antibodies in different participants) by Vi vaccines. While selection of the mAbs was carried out independently of the CD62L status of the plasmablast that was used for BCR sequencing, CD62L-positive plasmablasts were associated with a higher frequency of Vi PS antigen binding ( $\mathrm{p}<0.008$, Fig. 2G): $70 \%$ of the selected CD62L-positive plasmablasts were positive for Vi PS antigen binding, while only $40 \%$ of the CD62L-negative plasmablasts were positive for Vi PS antigen binding. Out of the 
96 mAbs, only two were found to bind TT (fig. S5). Combined, these data indicate that the selection strategy performed here was successful and could potentially be improved by incorporating CD62L status. To investigate maturation of the antibody response to $\mathrm{Vi}$, we examined the number of somatic hypermutations in the variable regions of the antibody heavy and light chains following prime and boost. The range of the mutation rate for post-prime mAbs was between 11-34 mutations and 2-47 mutations post-boost (fig. S6). This level of somatic hypermutation is consistent with recruitment of both naïve and memory B-cells shown in other vaccine regimes for influenza, streptococcus pneumoniae, etc (26-28). The mutation rate range was broader post-boost with the Vi-PS vaccine; however, only three monoclonal antibodies were selected from the post-prime group so median levels of mutations could not be compared between groups (fig S6).

Vi polysaccharide antibodies exhibit heterogeneous avidity and affinity maturation is not detected

To further characterize the binding and functional properties of Vi-specific mAbs, we first measured the avidity of each antibody to Vi PS antigen by BLI. Most mAbs avidities were within a range of $10^{3}-10^{5}\left(\mathrm{M}^{-1} \mathrm{~s}^{-1}\right)$ for on-rate and $10^{-2}-10^{-4}\left(\mathrm{~s}^{-1}\right)$ for off-rate. Overall, avidity measurements of the $53 \mathrm{mAbs}$, that were positive for binding to the polymeric Vi PS antigen, ranged from $10 \mu \mathrm{M}$ to $500 \mathrm{pm}$ (Fig. 3A) and $\mathrm{Vi}$ binding response magnitude showed moderate negative correlation with $\mathrm{K}_{\mathrm{D}}(\mathrm{r}=-0.60, \mathrm{p}<0.0001$, Fig. 3B), indicating a positive correlation with avidity. Only three antibodies were selected after prime with Vi-TT; these exhibited an avidity in the $1 \mu \mathrm{M}$ range, similar to that of mAbs isolated from the same participant post-boost (Fig. 3C and D). Similarly, polyclonal sera collected following Vi-TT prime and Vi-PS boost showed no evidence of 

maturation of $\mathrm{Vi}$ antibodies following repeat vaccination.

A.

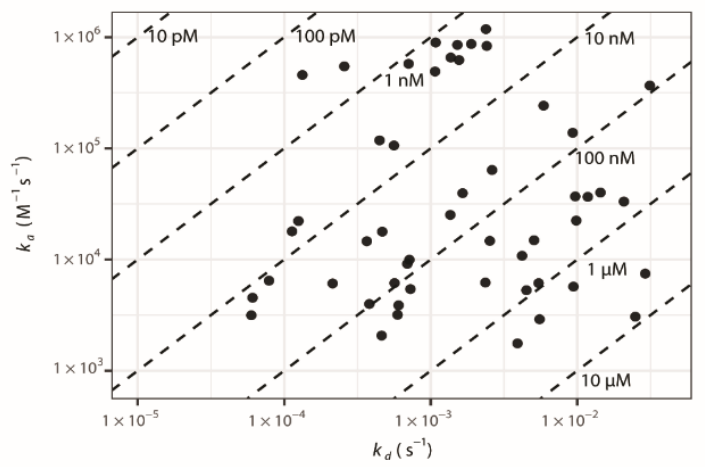

c.

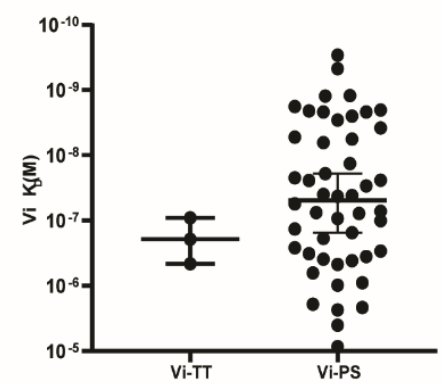

D.
230

231

232
B.
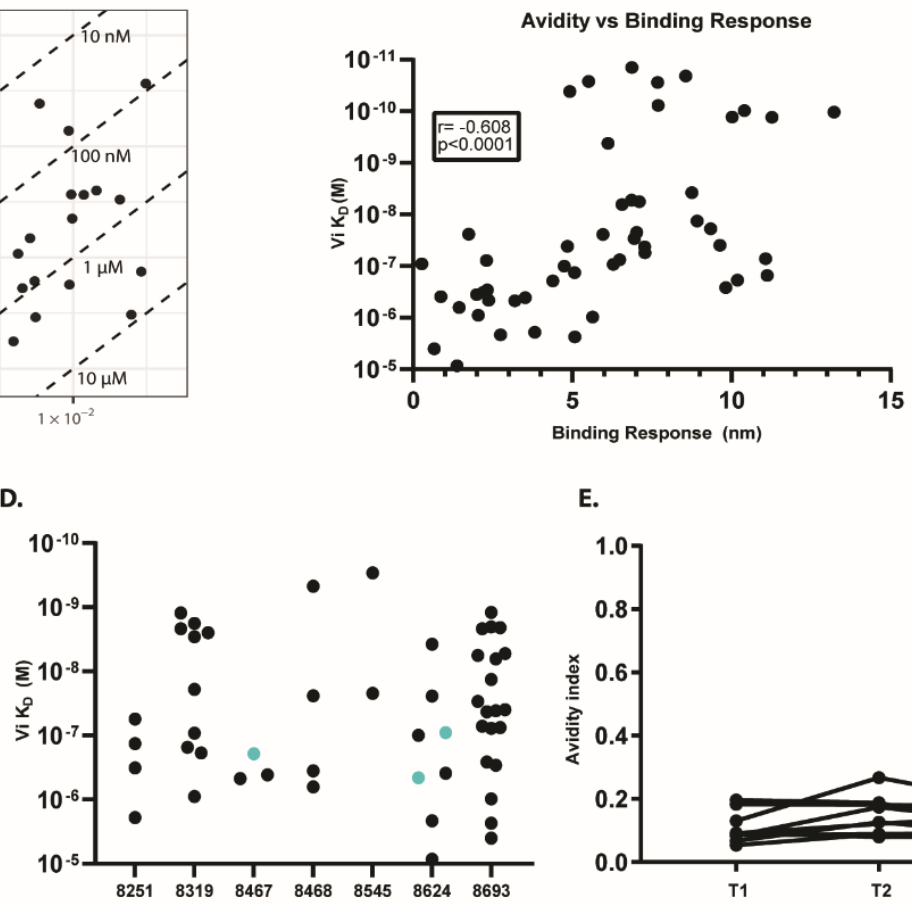

E.

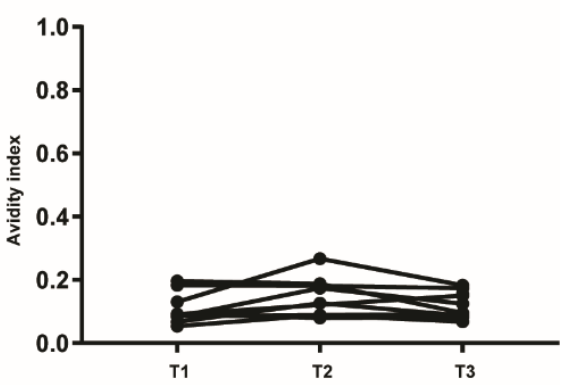

Fig. 3. No evidence for affinity maturation of Vi polysaccharide antibodies following repeat vaccination. A) Isoaffinity plot indicating avidity measurements for $\mathrm{mAbs}(\mathrm{n}=53)$ to polymeric Vi polysaccharide by BLI. Diagonal lines indicate the $\mathrm{K}_{\mathrm{D}}$ values shown. B) Spearman correlation of $\mathrm{Vi}$ antibody avidity and binding response to $\mathrm{Vi}$ antigen. Negative correlation represents decrease in $\mathrm{K}_{\mathrm{D}}$ which corresponds to an increase in avidity. C) Avidity measurements $\left(\mathrm{K}_{\mathrm{D}}\right)$ of Vi binding mAbs stratified by Vi-TT prime/ Vi-PS boost. D) Avidity measurements $\left(\mathrm{K}_{\mathrm{D}}\right)$ of Vi binding mAbs stratified by participant with blue indicating mAbs from prime time points. E) Total serum (T1 $\mathrm{n}=10, \mathrm{~T} 2 \mathrm{n}=8, \mathrm{~T} 3 \mathrm{n}=10$ ) was used to measure residual Vi antibody binding after NaSCN exposure for 15 minutes in ELISA, as a proxy for avidity measurements. The avidity index was calculated 
as Absorbance at $1 \mathrm{M} \mathrm{NaSCN} /($ Absorbance at $0 \mathrm{M} \mathrm{NaSCN}-$ Absorbance at $5 \mathrm{M} \mathrm{NaSCN}$ ). $\mathrm{T} 1=28$ days post-prime, $\mathrm{T} 2$ = before Vi-PS boost, $\mathrm{T} 3$ is 28 days after Vi-PS boost.

\section{$V i$ vaccines induce convergent B-cell responses with shared CDR3 characteristics}

Evidence of inter-donor B-cell sequence convergence in the anti-Vi immune response allowed for successful selection of $53 \mathrm{Vi}$-specific mAbs. To further investigate the level of convergence, we examined the sequence characteristics, avidity, and epitope binning of each group of convergent mAbs. We compared heavy chain sequences with the same $V_{H}$ gene segment among the seven donors from whom Vi PS antigen binding antibodies were successfully isolated. Using a cutoff for heavy chain CDR3 identity of $75 \%$ or greater (29), we identified five convergent groups of antibodies (G1-G5). Each convergent group included antibodies from two or more individual donors and six out of seven donors were represented in at least one group, suggesting convergent responses to the Vi PS antigen are common among vaccine recipients. Frequent use of $\mathrm{V}_{\mathrm{H}} 3-23$ and Vк3-11/ Vк3-15 was observed in convergent groups (Fig. 4A). Within groups that shared $\mathrm{V}_{\mathrm{H}}$ and $\mathrm{V}_{\mathrm{L}}$, all groups (G1-G5) shared a high level of CDRH3 sequence similarity (ranging from 76.5\% to $100 \%$ ) (Fig. 4B). Cross-competition of antibodies within each group provided further evidence that antibodies showing evidence of convergence target similar sites on Vi PS (Fig. 4C). In nearly all instances, no blocking was observed with a convergent pair when the higher avidity antibody bound after the lower avidity antibody (an example being lower avidity antibody AB-007972 versus higher avidity AB-007981 in G2) whereas when the high avidity bound first, the secondary antibody was blocked. Competition seen between mAbs of different convergent groups, in addition 
263 S7). Another possibility is that steric hindrance impedes binding to an adjacent but distinct site.

A.

\begin{tabular}{|c|c|c|c|c|c|c|c|}
\hline & $\begin{array}{l}\text { Participant ID, } \\
\text { Timepoint }\end{array}$ & $\mathrm{mAb}$ & IGHV & $\mathrm{V}_{\mathrm{H}} \mathrm{CDR} 3$ & IGLV & $V_{L}$ CDR3 & $\begin{array}{c}\text { Avidity }\left(K_{D}\right) \\
M\end{array}$ \\
\hline G1 & $\begin{array}{l}8624,-21 \\
8467,-21\end{array}$ & $\begin{array}{l}7977 \\
7994\end{array}$ & $3-23$ & $\begin{array}{l}\text { ARGRVATIRDFDYW } \\
\text { ARGRVATIRDFDYW }\end{array}$ & $3-11 k$ & $\begin{array}{l}\text { QQRNNWPLTF } \\
\text { QQRSNWPLTF }\end{array}$ & $\begin{array}{l}1.93 \mathrm{E}-07 \\
4.59 \mathrm{E}-07\end{array}$ \\
\hline G2 & $\begin{array}{l}8693, \mathrm{~V} 2 \\
8545, \mathrm{~V} 2\end{array}$ & $\begin{array}{l}7972 \\
7981 \\
\end{array}$ & $3-23$ & $\begin{array}{l}\text { AKSPVRGLIRDWYFDLW } \\
\text { AKSPARGTNRYWYFDLW }\end{array}$ & $3-20 K$ & $\begin{array}{l}\text { QQYGSSPWTF } \\
\text { QQYGSSPYTF }\end{array}$ & $\begin{array}{l}3.56 \mathrm{E}-07 \\
2.92 \mathrm{E}-10\end{array}$ \\
\hline G3 & $\begin{array}{l}8624, \mathrm{~V} 2 \\
8624, \mathrm{~V} 2 \\
8251, \mathrm{~V} 2 \\
8251, \mathrm{~V} 2\end{array}$ & $\begin{array}{l}7968 \\
7992 \\
7993 \\
8026\end{array}$ & $3-23$ & $\begin{array}{l}\text { AKGATGTIRAFDYW } \\
\text { ARGQTGTIRNFDYW } \\
\text { AKGHTGTIRDFDYW } \\
\text { AKGATATIRAFDYW }\end{array}$ & $3-11 k$ & $\begin{array}{l}\text { QQRSNWPITF } \\
\text { QQRSNWPITF } \\
\text { QQRSNWPLTF } \\
\text { QQRSNWPITF }\end{array}$ & $\begin{array}{l}1.34 \mathrm{E}-07 \\
2.43 \mathrm{E}-08 \\
2.14 \mathrm{E}-06 \\
3.22 \mathrm{E}-07\end{array}$ \\
\hline G4 & $\begin{array}{l}\text { 8693, V2 } \\
8319, \text { V2 }\end{array}$ & $\begin{array}{l}7983 \\
8000\end{array}$ & $3-74$ & $\begin{array}{l}\text { ARGSSAAAGTYYWFDPW } \\
\text { ARGRSAMAGTFYWFDPW }\end{array}$ & $3-15 k$ & $\begin{array}{l}\text { QQYNKWPQTF } \\
\text { QQYNKWPQTF }\end{array}$ & $\begin{array}{l}2.17 \mathrm{E}-09 \\
1.34 \mathrm{E}-08\end{array}$ \\
\hline G5 & $\begin{array}{l}8624, \mathrm{~V} 2 \\
8467, \mathrm{~V} 2\end{array}$ & $\begin{array}{l}7976 \\
7991\end{array}$ & $2-5$ & $\begin{array}{l}\text { AHRRGTGWYRG } \text { VDYW } \\
\text { AHRRGTGWYVGLDYW }\end{array}$ & $2-28 K$ & $\begin{array}{l}\text { MQALQTPCTF } \\
\text { MQALQTPGSF }\end{array}$ & $\begin{array}{l}4.70 \mathrm{E}-07 \\
1.00 \mathrm{E}-07\end{array}$ \\
\hline
\end{tabular}

Amino acid similarity: $\bigcirc$ Identical amino acids

- Similar amino acids (similar chemical characteristics)

Different amino acids

B. $\mathbf{G 1}$
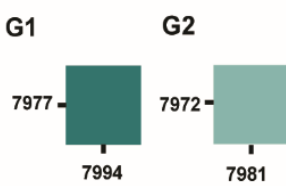

G3

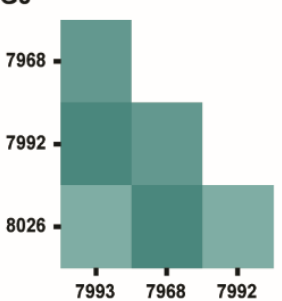

Similarity Index (\%)

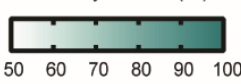

G4

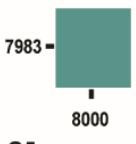

G5

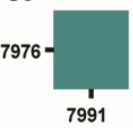

c.
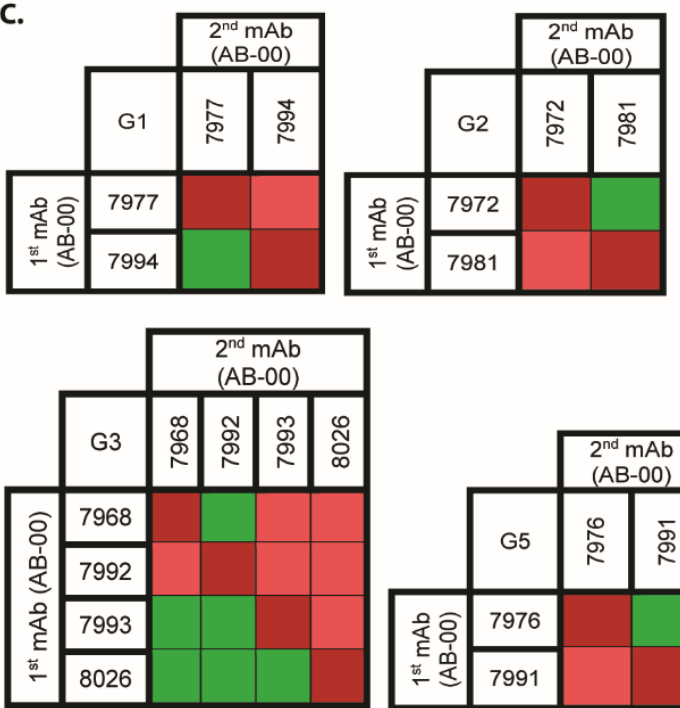
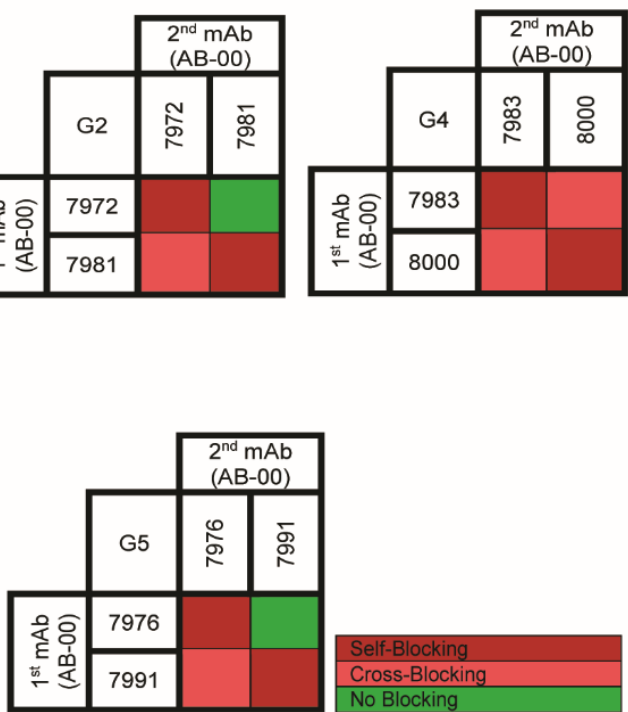

264

Fig. 4. Convergent development of Vi-specific antibody sequences among Vi vaccine recipients. A) Monoclonal antibodies $(n=12)$ from vaccinees with the same variable heavy chain gene segment were grouped and tested for similarity on the amino acid level by calculating the Levenshtein edit distance. Sequences from vaccinees with $\geq 75 \%$ identity were defined as a convergent group (G1-G5). Convergent groups based on variable heavy and light chain gene segment usage with participant, timepoint, $\mathrm{H}$ - and L-CDR3 sequence, and antibody avidity for Vi 
antigen. Bold black letters indicate identical amino acids, teal letters indicate amino acids with similar chemical characteristics, and pink letters indicate different amino acids. Chemical characteristics were defined by grouping amino acids based on electrically charged, polar uncharged, and hydrophobicity. B) Quantification of H-CDR3 sequence similarity by BLOSUM62 calculation of similarity index. C) Cross-competition of convergent antibodies within convergent groups by BLI, where $1^{\text {st }}$ mAb was bound to Vi to complete saturation before association with $2^{\text {nd }}$ mAb. Red indicates self-blocking, pink indicates cross-blocking, and green indicates no blocking.

\section{Monoclonal antibodies derived from Vi vaccination can target subdominant epitopes}

To further resolve the epitope specificity of Vi mAbs derived by vaccination, we employed a chemical modification to eliminate the immunodominant $O$-acetyl group at the $\mathrm{C} 3$ position and expose the polysaccharide backbone (Fig. 5A). The international standard (16/126) for Vi polysaccharide is $94.3 \% \mathrm{O}$-acetylated (30). Treatment with ammonium hydroxide $\left(\mathrm{NH}_{4} \mathrm{OH}\right)$ was titrated to determine the concentration at which the $O$-acetyl groups are completely removed. At 1.0M NH $\mathrm{MHH}_{4} \mathrm{OH}$, the Vi PS antigen was completely de-O-acetylated (Fig. 5B, fig. S8) leaving a new $\mathrm{C} 3{ }^{1} \mathrm{H}$ in its place. Out of $53 \mathrm{mAbs}, 47$ exhibited $\mathrm{C} 3 O$-acetyl-dependent epitope specificities, including AB-008053 where binding to the Vi PS antigen was reduced by over $75 \%$ upon de- $O$ acetylation (Fig. 5C and D, right). However, six mAbs (about $10 \%$ of the total pool) bound equally or with higher binding to de- $O$-acetylated Vi PS antigen as compared with native Vi PS antigen (Fig. 5D, left). Of those six mAbs, three displayed similar binding response and avidity to the native Vi PS and de- $O$-acetylated Vi PS antigens including AB-07988 (Fig. 5E, fig. S9A), indicating at least one subdominant, alternative epitope that is exposed (SEE) and independent of 
294 the $O$-acetyl group. In addition, three mAbs bound with greater binding and avidity to the de- $O$ 295 Vi PS antigen compared with the native Vi PS antigen including AB-08049 (Fig. 5F, fig. S9B), 296 indicating at least one subdominant epitope that is occluded (SEO) by the C3 $O$-acetyl and is less 297 solvent accessible. 
A.

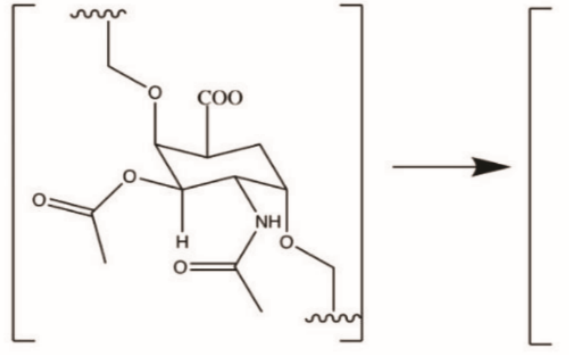

C.

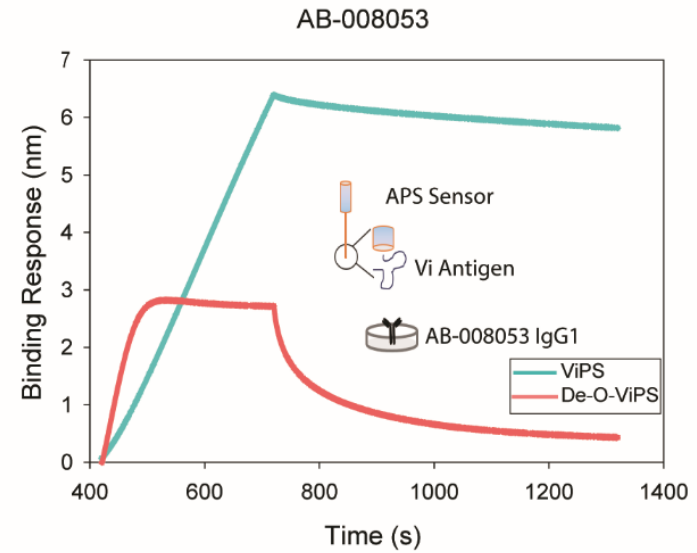

E.

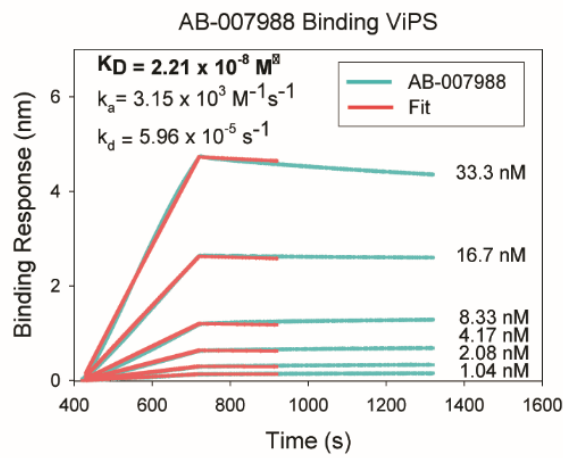

F.

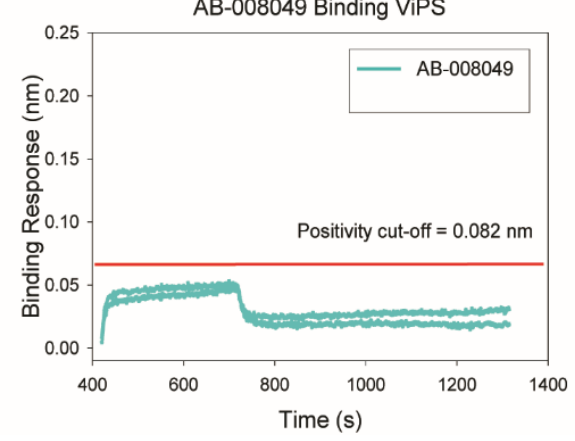

B.

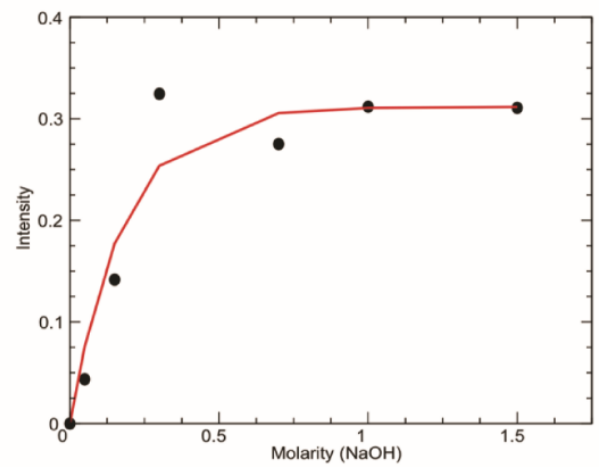

D.
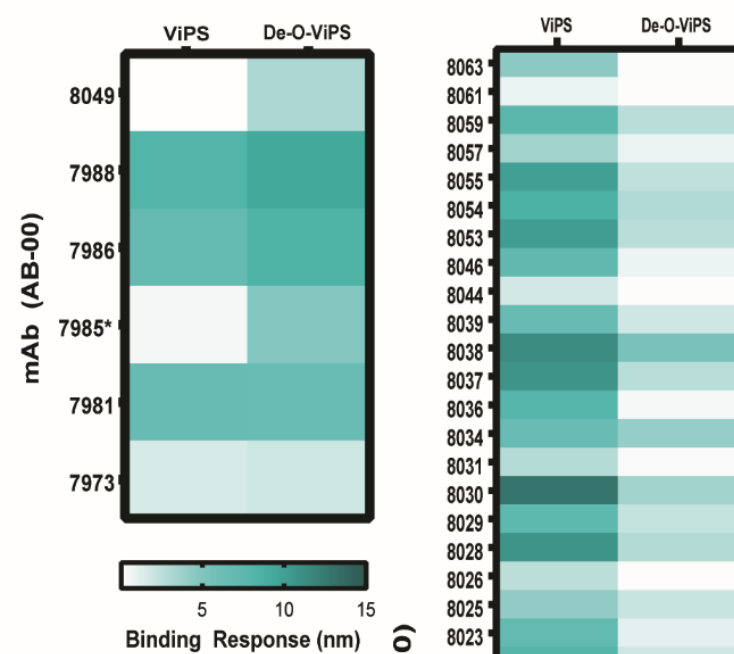

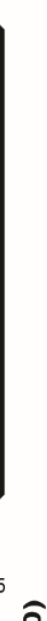

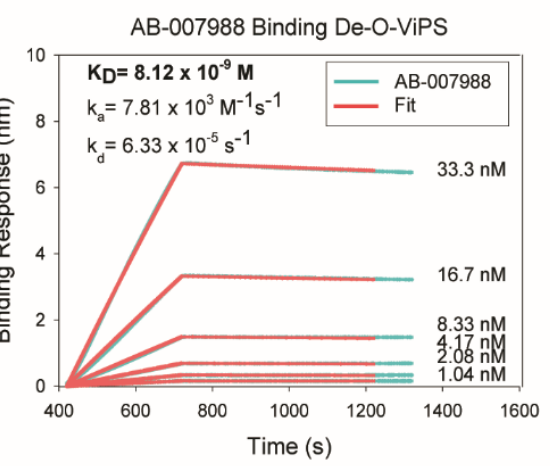

AB-008049 Binding De-O-ViPS

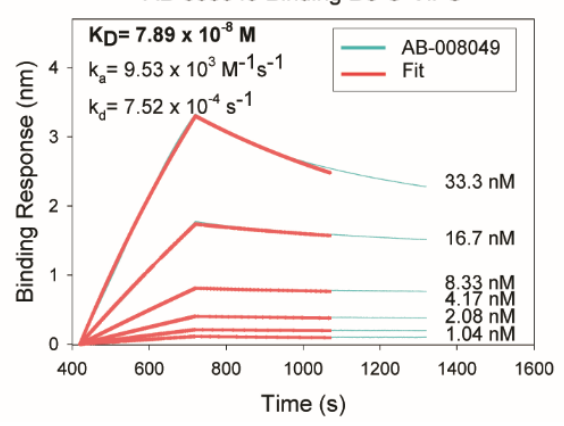

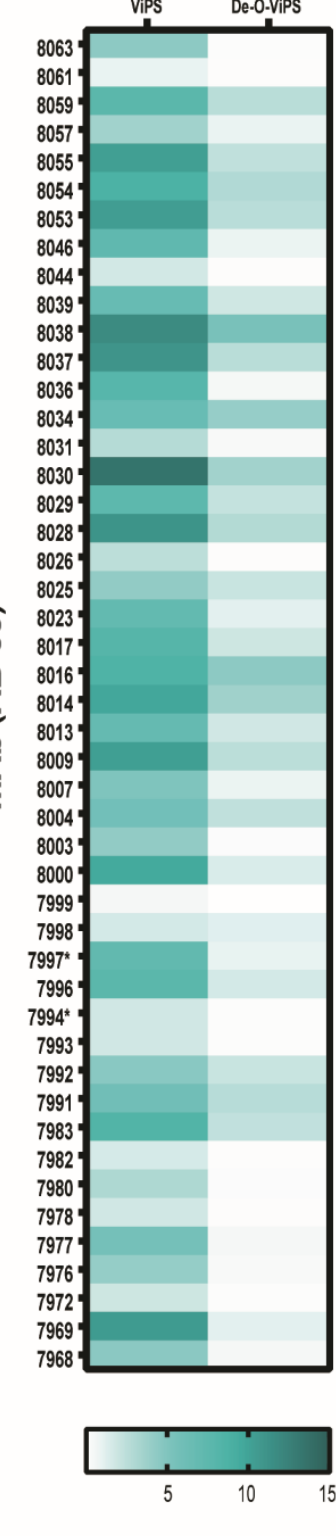

Binding Response (nm) 
301 structure of Vi polysaccharide monomer with $\mathrm{C} 3$ O-acetyl conversion to ${ }^{1} \mathrm{H}$ following $\mathrm{NH}_{4} \mathrm{OH}$

302 treatment. B) Increasing signal intensity of 2D NMR spectra centered around $1.9 \mathrm{ppm}\left({ }^{1} \mathrm{H}\right)$ and $30326.5 \mathrm{ppm}\left({ }^{13} \mathrm{C}\right)$ where new $\mathrm{C} 3{ }^{1} \mathrm{H}^{-13} \mathrm{C}$ bond appears as a function of increasing amounts of $\mathrm{NH}_{4} \mathrm{OH}$ 304 treatment $(n=7)$ of polysaccharide for de-O-acetylation. C) Binding sensorgram of AB-008053 IgG1 to ViPS and De-O-ViPS, where Vi is immobilized on biosensor and dipped into wells containing antibody. D) Heat map of binding response of all Vi binding mAbs ( $n=53)$ to ViPS and De-O-ViPS antigens. Asterisk indicates mAb was selected post Vi-TT prime. Left: mAbs with equal or greater binding to De-O-ViPS compared with ViPS (n=6). Right: mAbs with diminished binding to De-O-ViPS compared with ViPS (n=47). E, F) Example binding sensorgrams of mAbs interacting similarly (E) to ViPS (left) and De-O-ViPS (right) or better (F) to De-O-ViPS with corresponding avidity measurements indicated. $\Phi$ indicates lower antigen density used for antibody titration to reliably measure rate constants.

Competition experiments with the anti- $O$-acetyl antibody AB-008053 further defined the epitope specificities of these six alternate specificity mAbs. After Vi PS antigen binding was saturated by AB-008053, the further increase in binding signal mediated by AB-007986 and AB-007981 indicates that these mAbs have specificity for an epitope that is exposed (SEE) and non-adjacent to the $\mathrm{C} 3 \mathrm{O}$-acetyl group (Fig. 6A). AB-007988, which bound with equal binding and avidity to the Vi PS and de- $O$-acetylated Vi PS antigens as well (Fig. 5E), was slightly blocked by AB008053 in the competition assay (Fig. 6A). This suggests that AB-007988 binds an epitope adjacent to, but not dependent on, the $\mathrm{C} 3 O$-acetyl group, and that perhaps steric hindrance by $\mathrm{AB}$ - 
323008053 inhibited binding of AB-007988. This is confirmed by performing the competition in the 324 reverse orientation where AB-007988 associated first, followed by AB-008053 (fig. S10A). All 325 three mAbs that bound with higher binding and avidity to de- $O$-acetylated Vi PS compared with 326 the native Vi PS antigen were blocked by the anti- $O$-acetyl mAb AB-008053 (Fig. 6B), confirming 327 that the epitope specificity of these mAbs is shielded by the $\mathrm{C} 3 O$-acetyl group. Further, a cross328 competitive assessment of the six alternative epitope mAbs with each other confirmed that AB329007988 binds a distinct SEE epitope from the SEE epitope bound by AB-007986 and AB-007981 330 (Fig. 6C). In addition, there are two SEO epitopes, one that is only partially occluded by the $O$ 331 acetyl group and was bound by AB-007973, and one that is completely occluded by the $O$-acetyl 332 group and was bound by AB-008049 and AB-007985 (Fig. 6C). These experiments indicate that 333 AB-007973 and AB-007988 bind overlapping but non-identical epitopes where the AB-007988 334 epitope is more solvent accessible, and the AB-007973 epitope is slightly more occluded. 
A. Subdominant Epitopes Exposed (SEE)

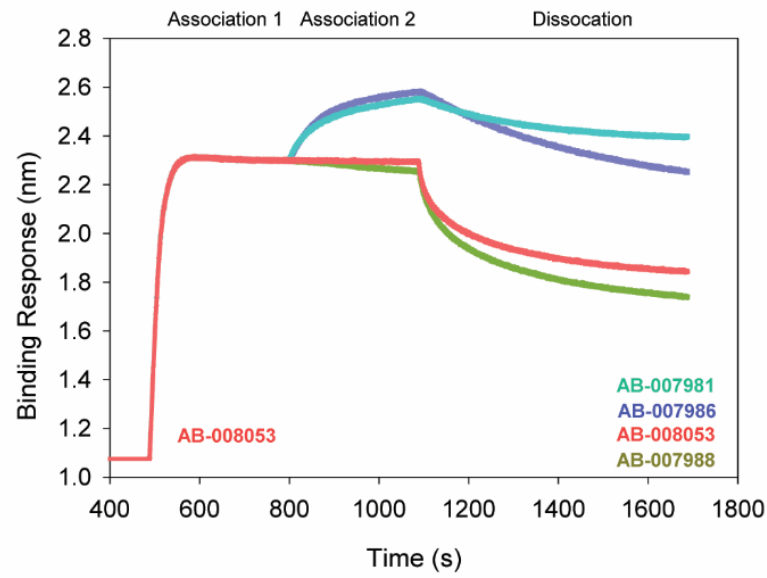

c.
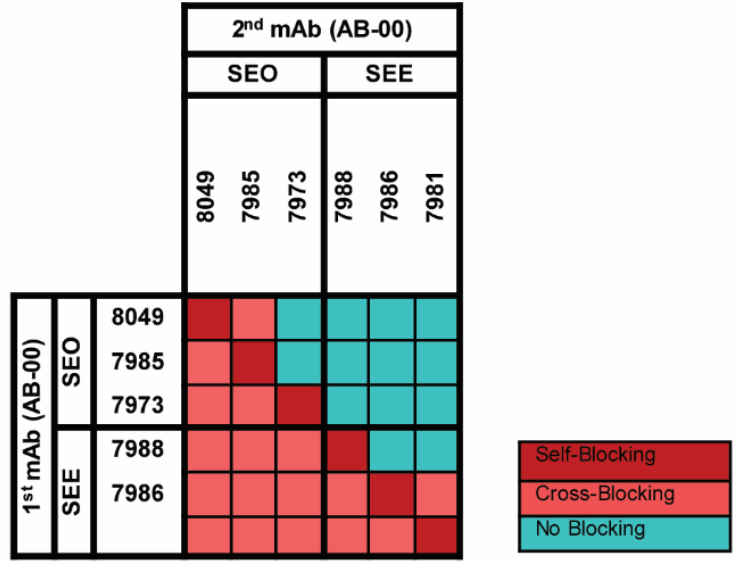
blocking and pink indicating cross-blocking.
B.

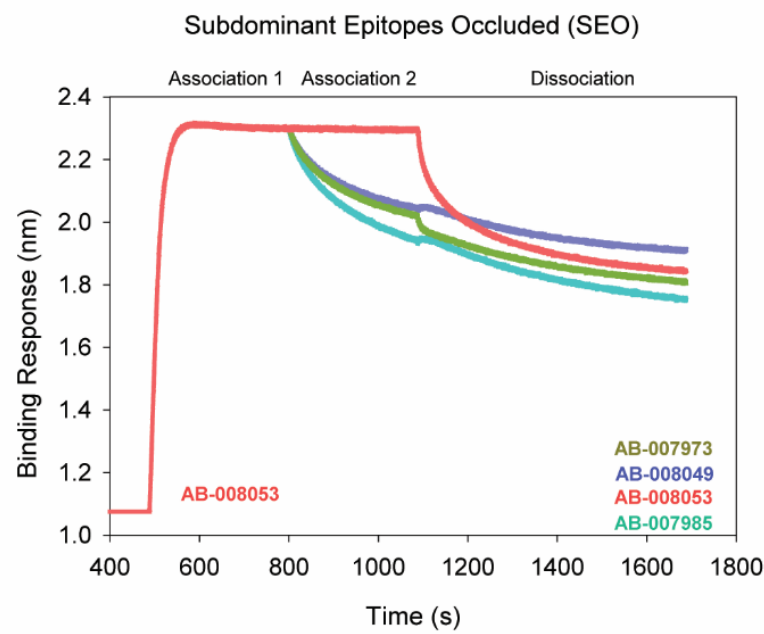

Fig. 6. ViPS monoclonal antibodies recognize exposed and occluded epitopes A) Competition sensorgram of O-acetyl targeting mAb AB-008053 as antibody 1 followed by antibodies $(n=3)$ targeting a subdominant but exposed epitope (SEE) as antibody 2 B) Competition sensorgram of O-acetyl targeting mAb AB-008053 as antibody 1 followed by antibodies $(n=3)$ targeting a subdominant and occluded epitope (SEO) as antibody 2 C) Cross-competition matrix of SEE $(n=3)$ and SEO $(n=3)$ targeting mAbs against each other with colored gradient, blue indicating no 
Vi antibodies mediate Fc-dependent effector functions

346 To identify the capacity of Vi binding antibodies to mediate Fc-dependent effector functions, we

347 evaluated the Vi-specific mAbs in in vitro monocyte phagocytosis and complement deposition 348 assays (fig S11 and 12). Vi-specific mAbs mediated a broad range of antibody-dependent 349 monocyte phagocytosis (ADMP) and antibody-dependent complement deposition (ADCD) 350 activity, with the mAbs specifically targeting an SEE epitope amongst the highest performers for 351 both ADMP and ADCD (Fig. 7A and B). On the contrary, mAbs that targeted an SEO epitope 352 occluded by the $\mathrm{C} 3 \mathrm{O}$-acetyl group performed poorly, likely due to the inaccessibility of the

354 mediate ADCD (Fig. 7C, r=0.51, p=0.0001).

The association rate (on-rate) of mAbs to Vi PS antigen had no impact on ADMP (fig. S13A, r=0.01, $\mathrm{p}=0.9452$ ) or $\mathrm{ADCD}$ (fig. $\mathrm{S} 13 \mathrm{~B}, \mathrm{r}=0.26, \mathrm{p}=0.063$ ); however, the dissociation rate (off-rate) of mAbs to Vi PS antigen was highly, negatively correlated (slower off-rate corresponds to higher function) with potency of ADMP (Fig. 7D, r=-0.50, p=0.0002) and ADCD (Fig. 7E, r=-0.74, $\mathrm{p}<0.0001$ ). ADMP was weakly correlated with antibody avidity (Fig. 7D, r=0.38, p=0.005); however, ADCD was highly correlated with antibody avidity (Fig. 7E, r=-0.76, p<0.0001). In fact, in the current experimental set-up, Vi mAbs with avidity lower than $500 \mathrm{nM}$ did not mediate ADCD with Vi PS antigen-coated beads (Fig. 7E). These results indicate that antibodies targeting any epitope that is accessible, not merely the immunodominant $\mathrm{C} 3 \mathrm{O}$-acetyl group, can mediate Fc-effector function in a manner dependent on the antibody-antigen off-rate. 
A.

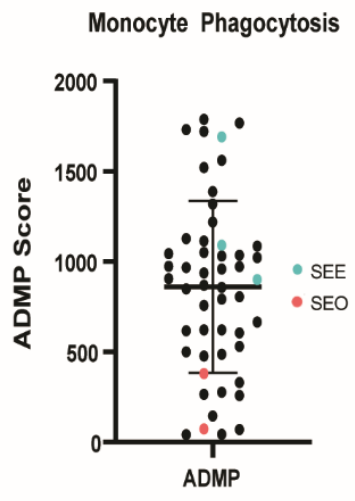

B.

Complement Deposition

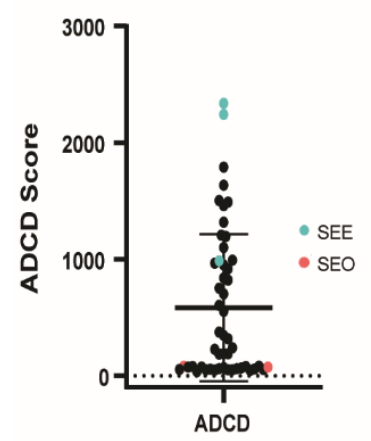

c.

C. $\quad A D M P$ vs ADCD

D.

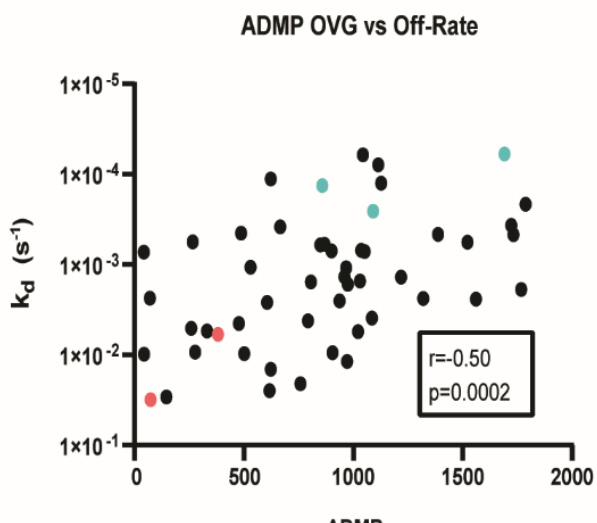

E.
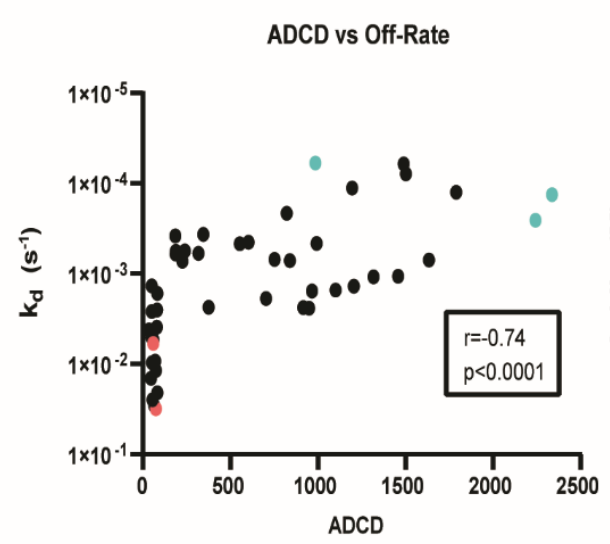

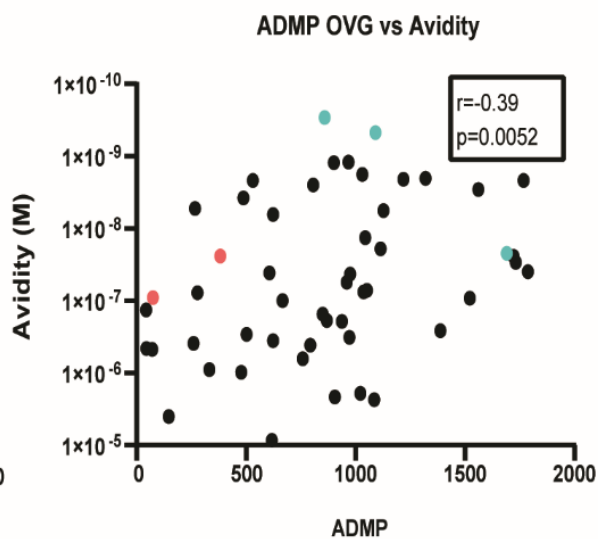

$A D C D$ vs Avidity

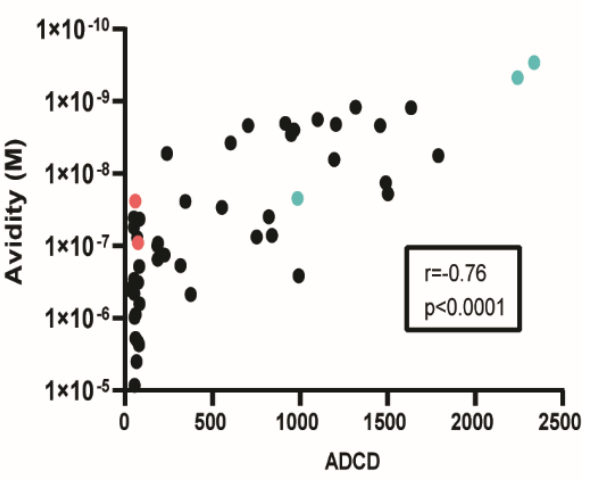

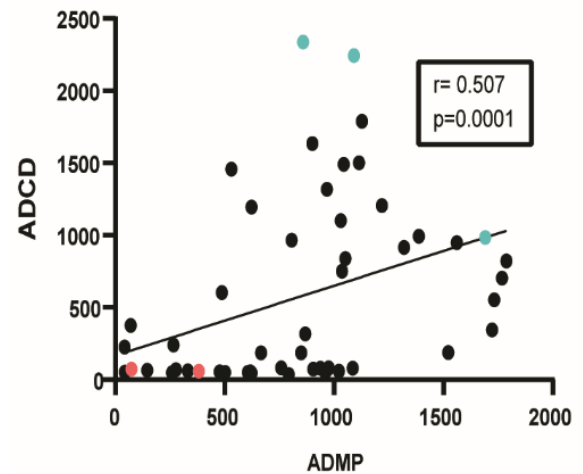

367 Fig. 7. ViPS antibodies mediate non-neutralizing Fe effector functions which are highly 368 associated with slow antibody dissociation rates A) Antibody-dependent monocyte 369 phagocytosis (ADMP) scores and B) antibody-dependent complement deposition (ADCD) scores 370 for Vi binding monoclonal antibodies $(\mathrm{n}=53)$ where blue indicates antibodies that bind to 371 subdominant exposed epitopes ( $\mathrm{SEE}, \mathrm{n}=3$ ) and pink indicates antibodies that bind to subdominant 
372 occluded epitopes (SEO, $n=3$ ). C) Spearman correlation of ADMP and ADCD scores for Vi 373 monoclonal antibodies ( $n=53)$. (D) Spearman correlations of ADMP with antibody off-rate (left) 374 and avidity (right). E) Spearman correlations of ADCD with antibody off-rate (left) and affinity 375 (right). Negative correlations represent decreases in off-rate and $\mathrm{K}_{\mathrm{D}}$ which corresponds to 376 increases in avidity.

377

378

379

\section{Discussion}

Recent analyses of immune correlates for typhoid Vi PS vaccines suggest that protection from typhoid is mediated by high titers of high avidity IgG and IgA antibodies in combination with antibody-mediated innate immune cell function $(12,16,17)$. Although Vi antibodies correlate with protection against typhoid, the properties of the vaccine-induced humoral immune response to $\mathrm{Vi}$ remain unknown. In this study, we isolated plasmablasts from individuals primed and boosted with Vi vaccines and evaluated the repertoire, avidity maturation, epitope specificity, and functionality of Vi mAbs. Our findings highlight that Vi vaccination elicits convergent, functional antibodies that target both immunodominant and subdominant Vi epitopes, and that ability to mediate Fc effector function is largely associated with antibody dissociation kinetics.

After both prime (Vi-TT) and boost (Vi-PS), the predominant antibody subclass expressed by Bcells was IgG1, the most common antibody subclass in serum. A Vi-PS boost resulted in an increase in IgG2-expressing plasmablasts likely from new marginal zone or B1 B-cells induced directly by the unconjugated, T-independent polysaccharide. Previously it has been reported that polysaccharides are likely to induce an $\mathrm{IgG} 2$ response (31-33), which has also been observed for the Vi-PS vaccine (16). The number of IgG3 sequences detected in this study is lower than would 
be expected based on antibody data (16). Responses evaluated following Vi-PS would include boosting of existing memory B-cells induced by the conjugate prime as well as newly activated Bcell clones, consistent with the broad range of somatic hypermutations in the antibody variable regions (range=2-47). Therefore, while the prime-boost strategy allows for a robust humoral response to be generated toward $\mathrm{Vi}$, this strategy does make it difficult to determine whether the Vi-PS vaccine response evaluated here is a typical response for Vi-PS immunization. The primeboost strategy does allow the direct comparison of repertoires within the same individual. Here, we highlighted four of the IgHV genes for which a difference in frequency was observed between the Vi-TT and Vi-PS vaccines. IgHV3-23 is one of the most commonly observed genes (34) and antibodies using this region have previously been described to recognize other polysaccharides such as the Haemophilus influenzae type b (Hib) polysaccharide (35). Allelic variances in IgHV323 were also shown to influence antibody binding and effector function towards this antigen (35).

Using the selection criteria of convergence, persistence, and expansion, we isolated 96 mAbs of which 52 were positive for binding the $94.3 \% O$-acetylated Vi PS antigen, with one additional binding to de- $O$-acetylated Vi PS antigen only, for a total of 53. Only two mAbs bound to TT; therefore, the remaining $41 \mathrm{mAbs}$ have either an unidentified antigen-specificity or the affinity of these antibodies for $\mathrm{Vi}$ is too low to measure by BLI. The mAbs which had measurable binding responses for $\mathrm{Vi}$ exhibited a range of $\mathrm{K}_{\mathrm{D}}$ from around $10 \mu \mathrm{M}$ to around $500 \mathrm{pM}$; however, the measurement of $\mathrm{K}_{\mathrm{D}}$ is representative of avidity not true affinity due to the size of the Vi polymer and binding by full IgG molecules. Future studies to measure affinity would require digestion of mAbs into single Fab fragments and synthesizing the Vi minimal epitope, shown to be a hexamer (36), rather than using the native Vi PS antigen which contains greater than 10,000 units (37). Therefore, the measured avidity must be higher than the true affinity of these mAbs, which are 
likely to be near the lower range of $10 \mu \mathrm{M}$, similar to most other isolated polysaccharide mAbs against other pathogens $(38,39)$. There was no evidence of affinity maturation at the population level, also reported in a CHIM study following Vi vaccination (16), and there is unlikely to be affinity maturation occurring on the individual level within a clonal lineage. Here we selected one representative $\mathrm{mAb}$ from each expanded or persistent family; therefore, the changes within clonal lineages cannot be examined. However, these results are not unexpected as boosting with unconjugated $\mathrm{Vi}$ polysaccharide, a $\mathrm{T}$ cell independent antigen, is unlikely to drive affinity maturation $(40,41)$.

Naturally occurring $S$. Typhi isolates exhibit Vi PS where 60-90\% of monomers are $O$-acetylated (21), and currently licensed Vi based vaccines require a threshold of $>52 \% O$-acetylation (23). Previous studies have established that vaccination with highly $O$-acetylated Vi still induces low level polyclonal antibody responses to other unknown epitopes on the polysaccharide backbone $(20,22)$, but the functional relevance of these subdominant responses, if any, has not been described. Here we show that around 10\% of the selected Vi PS plasmablast pool are specific for subdominant, non- $O$-acetyl epitopes, and that these antibodies appear post-prime, post-boost, and in more than half of the donors, indicating that these responses are common. However, whether these antibodies arise due to new epitope exposure via Vi conjugation to TT, which has been shown to change the conformation and flexibility of other vaccine antigens, is unknown $(42,43)$. We show that although the $O$-acetyl group is the immunodominant epitope, antibodies arise that target at least four distinct, alternative epitopes, two that are subdominant but solvent exposed (SEE) and two that are completely or partially occluded from the surface (SEO). The level of convergence and competition between Vi binding mAbs suggests that likely only a small number of overlapping and adjacent epitopes exist on the Vi PS. Possible epitope specificities are presumably limited 
441 because of the nature of the antigen as a homopolymer with a small repeating monomer. Epitope 442 mapping using additional site-directed modifications of the polysaccharide would define the 443 epitope specificities of these mAbs and determine whether the mAb binding sites are identical or 444 are competing by steric hindrance due to recognition of overlapping epitopes.

We observed that mAbs targeting the subdominant exposed epitopes were among the highest performers for ADMP and ADCD, indicating that responses toward subdominant regions may be functional in preventing or controlling typhoid fever. However, mAbs targeting the subdominant epitope occluded by $O$-acetyl groups performed poorly for ADMP and ADCD likely due to the inaccessibility of the epitope for binding. Partial de-O-acetylation of Vi ( 45-60\%) increased immunogenicity of $\mathrm{Vi}(20)$ by increasing the flexibility of the polysaccharide backbone (22), and de- $O$-acetylation also exposes new epitopes. We found that antibodies to these exposed subdominant epitopes (SEE) mediated potent antibody-dependent Fc effector functions providing rationale to test the role of these antibodies in protection against or control of $S$. Typhi infection.

Properties that influence antibody-mediated effector functions include epitope-paratope interactions as well as Fc-FcR interactions. We found that potency of monocyte phagocytosis and complement deposition were significantly correlated with antibody off-rate, but not with on-rate, consistent with a prior study (44) and distinct from neutralization which was linked to association rates $(45,46)$.

This study has several limitations. The focus of the antibody kinetics and functional 461 analyses are limited to Vi IgG1 mAbs, without analysis of additional isotypes and subclasses. 462 Given that a large proportion of antibodies mounted against Vi PS post-vaccination with Vi-PS 463 are of the IgG2 subclass and that allosteric changes can influence binding kinetics (47) (48), further 
studies with the natural antibody isotype and subclass are needed. Additionally, since Vi PS IgA correlated with protection in a CHIM to study typhoid (16), an assessment of the Ig $\mathrm{A}^{+}$plasmablast pool and characterization of IgA specificity and function is warranted. Other limitations of this study include the small number of participants from which plasmablasts were isolated $(n=8)$ and the small number of prime time-point antibodies and prime-boost paired samples to assess affinity maturation. However, the presence of high avidity antibodies following the boost and the finding that function is dependent on maturation of the antibody off-rate provides evidence that boosting may elicit more kinetically and functionally mature antibodies. High avidity antibodies with antibody $\mathrm{Fc}$ effector functions correspond with protection from disease in a typhoid CHIM (16, 17). Serum bactericidal activity did not correlate with protection; however, the role of complement activation as part of a polyfunctional response is not fully understood (49). There is room to improve on the efficacy of the Vi-TT vaccine regimen to move from $82 \%$ and $85 \%$ efficacy in Nepal and Bangladesh, respectively $(13,50)$ to an ideal of $100 \%$ in diverse populations, including immunocompromised individuals. The results from this current study provide rationale to test whether a boost immunization provides better protection than a single dose vaccination alone to achieve maximum efficacy in all populations. While the Vi-PS boost in this study enriched Vispecific plasmablasts to study Vi-specific immunity, likely a boost with Vi-TT would provide the most robust affinity maturation while maintaining the critical memory B-cell pool.

In summary, we identified that antibodies to both dominant and subdominant epitopes mediated in vitro phagocytosis and complement deposition. Moreover, functional responses correlated with antibody dissociation rate from Vi. Broadening the antibody epitope targeting and Fc mediated effector functions for $S$. Typhi Vi is a promising strategy for improving Vi vaccine regimens with higher efficacy in different populations. 
Materials and Methods

489

490

491

492

493

494

495

496

497

498

499

500

501

502

503

504

505

506

507

508
Study design

The objective of our study was to characterize the human antibody response induced by a primeboost vaccination strategy against Salmonella Typhi virulence factor Vi polysaccharide, and to test the hypothesis that diverse and subdominant epitope specificities could mediate antibody Fc effector functions. We sorted and sequenced plasmablasts from vaccinated individuals $(n=8)$ after prime and boost and selected a pool $(n=96)$ of sequences to express as monoclonal antibodies. We examined the convergence in B-cell responses by examining $\mathrm{V}$ gene usage and CDR3 characteristics between vaccinees. Using different forms of the Vi polysaccharide antigen $(O$ acetylated and de- $O$-acetylated), we defined the specificity of vaccine-derived monoclonal antibodies and the avidity maturation of these antibodies following prime and boost. Finally, we investigated the ability of these antibodies to mediate Fc-effector functions in cell-based assays and established the relationship between Fc-function, specificity, and avidity.

Sample Collection: We analyzed twelve participants, from a completed clinical trial (12), who were primed with a Vi-TT vaccine (Typbar-TCV, Bharat Biotech, Hyderabad, India). PBMCs were acquired before immunization and 7 days post-immunization. Serum was available preimmunization and 28 days post-immunization with Vi-TT. Five participants were orally challenged with $S$. Typhi, 28 days after receiving the initial immunization. Challenge did not increase antibody responses toward $\mathrm{Vi}(51)$.

All participants received a boost with a plain Vi-PS vaccine (TYPHIM Vi, Sanofi Pasteur, Lyon, France). The Vi-PS boost was administered between 19 and 23 months after the initial Vi-TT 
509 immunization. PBMCs were acquired pre-immunization, 7 days post-immunization and serum was 510 available 28 days post-immunization with Vi-PS.

All volunteers provided written informed consent before enrollment. The study protocol was approved by the sponsor (University of Oxford), the South Central Oxford A Ethics Committee

514 (14/SC/1427), and the Medicines and Healthcare Products Regulatory Agency (Eudract 2014515 002978-36).

Single cell BCR sequencing

PBMC samples were selected for BCR sequencing based on sample availability. Single CD62L'

519 or $\mathrm{CD}^{2} \mathrm{~L}^{+}$plasmablasts (CD19+CD20-CD38hiCD27+CD3-CD14-IgA-IgM-IgD-) were individually sorted into wells of 384-well plates using a FACSAria Fusion (BD Biosciences, USA). Generation of barcoded cDNA, PCR amplification, and sequencing of IgG genes were performed as described previously (52) with the following modifications: biotinylated Oligo(dT) and RT maxima H- (Fisher Scientific Company) were used for reverse transcription, cDNA was

524 extracted using Streptavidin C1 beads (Life Technologies), and DNA concentrations were determined using qPCR (KAPA SYBR® FAST qPCR Kit for Titanium, Kapabiosystems). V(D)J assignment and mutation identification was performed using a variant of SoDA (53).

Variable heavy and light chain DNA sequences were synthesized and cloned into the respective human IgG1 heavy chain or endogenous light chain expression plasmid. The matched pair of 
531 heavy and light chain plasmids were transiently co-transfected into HEK-293 cells. Antibody

532 supernatants were harvested 7 days post-transfection, purified using Protein A affinity

533 chromatography and filtered through a $0.2 \mu \mathrm{m}$ filter. The final elution buffer for all antibodies

534 was $100 \mathrm{mM}$ HEPES, $100 \mathrm{mM} \mathrm{NaCl}, 50 \mathrm{mM} \mathrm{NaOAc}, \mathrm{pH}$ 6.0. Nomenclature for isolated

535 antibodies includes AB-00 followed by a four number string. For graphing purposes, AB-00 may

536 be omitted.

\section{Vi-biotinylation and bead coupling}

539 The International Standard for Citrobacter freundii Vi PS (NIBSC 12/244) with a 94.3\% O540 acetylation level was biotinylated by Expedeon (Cambridge, UK). A biotin binding assay (HABA) 541 was carried out to confirm successful biotinylation. It was estimated that each Vi PS molecule 542 contained 14 biotins after the modification procedure.

543 PE-neutravidin beads (Life technologies) were coated with Vi by incubating $2.5 \mu \mathrm{g}$ of biotinylated 544 Vi with $10 \mathrm{ul}$ of beads for a duration of 2 hours at 37 degrees. Beads were washed twice with $1 \mathrm{ml}$ of PBS 5\% BSA (microcentrifuged at 13000rpm for 2 minutes) and taken up in 1ml of RPMI for each 10ul of original bead volume. Coupled beads were used for functional measurements.

\section{BioLayer Interferometry (BLI)}

549 BLI measurements were collected with ForteBio Octet RED384 instruments. Kinetics of Vi mAbs 550 binding to $\mathrm{Vi}$ PS or de- $O$-Vi PS were analyzed by BLI as previously described (16) with 551 modifications. Briefly, native Vi PS antigen or de- $O$-acetylated Vi PS $(5 \mu \mathrm{g} / \mathrm{ml})$ was immobilized 552 to ForteBio aminopropylsilane (APS) biosensors via hydrophobic interaction, followed by 
washing sensors with 10X Kinetics Buffer (10X KB) (ForteBio, USA) to coat unoccupied sensor area and minimize non-specific interactions. A baseline was established in $1 \mathrm{X} \mathrm{KB}$, and then Viloaded sensors were dipped into wells containing $\mathrm{Vi}$ mAbs (in $1 \mathrm{X} \mathrm{KB}$ ) to monitor $\mathrm{mAb}$ association. Dissociation was monitored by dipping Vi mAb associated sensors back into the 1X KB wells used to collect the baseline time-course. Each mAb was titrated 7 places starting at 10 $\mu \mathrm{g} / \mathrm{ml}$, 2-fold. Parallel blank sensors that were also washed in 10X KB and dipped in Vi mAbs were used to subtract out non-specific interactions. At least 4/7 reference subtracted binding curves were globally fit using ForteBio Data Analysis 10.0 software to determine antibody binding response $(n m)$, on-rate $\left(k_{a}\right)$, off-rate $\left(k_{d}\right)$, and observed avidity $\left(K_{D}\right)$. Fitting window was adjusted to minimize effects of upward drift/rebinding in the dissociation phase.

Competition experiments were conducted by immobilizing Vi PS antigen $(5 \mu \mathrm{g} / \mathrm{ml})$ to APS biosensors at very low density (0.01 nm loading threshold). After washing with $10 \mathrm{X} \mathrm{KB}$, sensors were dipped into wells containing $10 \mu \mathrm{g} / \mathrm{ml}$ (in $1 \mathrm{X} \mathrm{KB}$ ) of antibody 1 to bind to complete saturation. Sensors were then dipped into wells containing a second Vi mAb at $10 \mu \mathrm{g} / \mathrm{ml}$ (in $1 \mathrm{X}$ $\mathrm{KB})$. Lastly, sensors were dipped back into wells containing $1 \mathrm{X} \mathrm{KB}$ to allow dissociation. No blocking is described as an increase in binding signal upon dipping into antibody 2 . Blocking is described as a flat line or decrease in binding signal upon dipping into antibody 2.

\section{ELISA}

Serum Vi IgG antibodies were quantified using the VaccZyme Human Anti-S typhi Vi IgG ELISA kit (VaccZyme, Birmingham, UK). Specificity of mAbs was determined using the VaccZyme Human Anti-S typhi Vi IgG ELISA kit and the tetanus toxoid IgG ELISA kit (VaccZyme, 
Birmingham, UK). Total serum antibody data was published previously as part of the main study (12).

Total serum avidity was measured using a modified Vi ELISA in combination with NaSCN. Nunc Maxisorp U-bottom plates were coated using streptavidin (Thermo Scientific, 43-4301) at $2 \mu \mathrm{g} / \mathrm{ml}$ overnight at 37 degrees, followed by a 1 hour incubation with biotinylated Vi at $2 \mu \mathrm{g} / \mathrm{ml}$ for 1 hour at room temperature (RT). The plate was blocked with PBS $1 \%$ BSA for 1 hour at RT. Serum dilutions were chosen to ensure that the binding of the samples was in the linear phase of their titration curve. Samples from the same person were tested on one plate. Serum samples, diluted in PBS, $1 \%$ BSA, 30\% Brij 35, were incubated for 1 hour at 37 degrees and incubated with several concentrations of $\mathrm{NaSCN}(5 \mathrm{M}, 1 \mathrm{M}, 0.5 \mathrm{M}, 0.25 \mathrm{M}, 0.125 \mathrm{M}$, and $0 \mathrm{M})$ for 15 minutes at $\mathrm{RT}$. $\mathrm{IgG}$ antibodies bound to the plate were detected with Mouse monoclonal anti-human IgG Fc-Alkaline Phosphatase (Abcam, ab99764), incubated for 1 hour at 37 degrees. Residual binding was detected using the combination 4-Nitrophenyl Phosphate (pNPP) Liquid Substrate System (Sigma Aldrich, N7653) and a $3 \mathrm{M} \mathrm{NaOH}$ stop solution.

The avidity index of each sample was calculated as a percentage of residual binding: $\mathrm{xM} /(0 \mathrm{M}-$ $5 \mathrm{M})$, where $\mathrm{x}$ was a concentration of $\mathrm{NaSCN}$ in between $0 \mathrm{M}$ and $5 \mathrm{M}$.

\section{De-O-acetylation of Vi polysaccharide}

Vi PS was de- $O$-acetylated using a method from Hitri et al (22) adapted from the method described by Szu et al (20). Briefly, one vial of the International Standard for Vi PS NIBSC 12/244 was rehydrated in water $(1 \mathrm{mg} / \mathrm{ml})$ and was diluted in airtight tubes with $10 \mathrm{M}$ ammonium hydroxide $\left(\mathrm{NH}_{4} \mathrm{OH}\right)$ to a final concentration of $50 \mathrm{mM}, 150 \mathrm{mM}, 300 \mathrm{mM}, 500 \mathrm{mM}$, and $700 \mathrm{mM}$ for partial 
de- $O$-acetylation, and $1 \mathrm{M}$ or $1.5 \mathrm{M} \mathrm{NH} 4 \mathrm{OH}$ for full de- $O$-acetylation. Samples were capped and incubated at $37^{\circ} \mathrm{C}$ for 18 hours. Solvent was evaporated and the de- $O$-acetylated Vi PS (de- $O$-Vi PS) pellet was re-hydrated in water overnight before use. For NMR experiments, Vi PS was rehydrated before and after de- $O$-acetylation with deuterated water $\left(\mathrm{D}_{2} \mathrm{O}\right)$.

\section{Nuclear Magnetic Resonance Spectroscopy (NMR)}

NMR was performed on a Bruker 16.4 Tesla spectrometer (Bruker, USA) with a BBO room temperature probe. Before measurement, individual samples were exchanged/lyophilized two times, each time resuspending the polysaccharide in $600 \mu \mathrm{L} \mathrm{D} 2 \mathrm{O}$. The $O$-acetylation level of control (untreated), $0.05 \mathrm{M}, 0.15 \mathrm{M}, 0.3 \mathrm{M}, 0.7 \mathrm{M}, 1.0 \mathrm{M}$, and $1.5 \mathrm{M} \mathrm{NH}_{4} \mathrm{OH}$-treated Vi PS solutions were investigated by 2D-dimensional 1H-13C Heteronuclear Single Quantum Coherence (HSQC) spectroscopy analyses. Quantification of the $N$ - and $O$-acetyl signals were possible in the ${ }^{1} \mathrm{H}-{ }^{13} \mathrm{C}$ HSQC as they are well-resolved. The intensity of the $N$ - and $O$-acetyl crosspeaks ( $\sim 1 \mathrm{H}$ at $1.9 \mathrm{ppm})$ was used to determine the ratio of $O$ - to $N$-acetylation. ${ }^{1} \mathrm{H}-{ }^{13} \mathrm{C}$ HSQC two-dimensional experiments were performed using the hsqccetgpsisp2.2 pulse sequence, with a $1 \mathrm{~J}(\mathrm{CH})$ coupling constant of $145 \mathrm{~Hz}$. Processing was done using Topspin 4.0.1 (Bruker, USA).

\section{Antibody-Dependent Monocyte Phagocytosis (ADMP)}

The capacity of mAbs (concentration: $1 \mu \mathrm{g} / \mathrm{ml}$ ) to mediate ADMP was performed as previously described in THP1 cells (54). The phagocytic score for each sample was determined by multiplying the \% of bead-positive cells with the GMFI (geometric mean fluorescence intensity) of the bead- 
618 positive cells. Debris and doublets were removed by manual gating before carrying out these 619 calculations.

622 ADCD was performed as previously described (55), with the following modifications. MAbs $623(1 \mu \mathrm{g} / \mathrm{ml})$ were incubated with antigen coated beads for 15 minutes at 37 degrees and then with 624 complement (low-tox guinea pig complement (Cedarlane, CL4051)) for 15 minutes at 37 degrees.

625 The plate was centrifuged at $1000 \mathrm{~g}$ for 3 minutes at 4 degrees, plates were washed with $200 \mu 1 /$ well 626 of PBS with $15 \mathrm{mM}$ EDTA, incubated with FITC-conjugated Goat IgG Fraction to Guinea Pig 627 Complement C3 (MP Biomedical, 855385) for 20 minutes, and the wash step was repeated.

628 Complement deposition was assessed by determining the GMFI of anti-C3 intensity on PE629 positive beads (BD Fortessa X20). Doublets and debris were removed by manual gating before 630 carrying out these calculations.

\section{Quantification and Statistics}

632 The sequence similarity index of CDRH3 sequences in convergent antibody groups was calculated 633 by EMBOSS-Needle using pairwise alignment and BLOSUM62 (BLOcks Subsitution Matrix 62) 634 with residue-specific and hydrophilic gap penalties (open gap penalty $=10$, extended gap penalty 635 0.5). For the selection of antibodies for protein expression based on convergence, the BLOSUM62 636 similarity was calculated for pairs of antibodies from different donors. Putative convergent 637 antibodies were required to have greater similarity than that observed between pairs of unrelated 638 antibodies (56). 
CDR3 lengths were examined by the Shapiro-Wilk normality test R software [version 3.6.1]. The

640 relationship between CD62L expression on plasmablasts and antibody Vi-specificity were

641 evaluated by Fisher's exact test and the relationship between the functional and kinetic

642 measurements were evaluated by Spearman correlation analysis in SAS software [Version 9.4 of

643 the SAS System Copyright @ 2002-2012 by SAS Institute Inc., Cary, NC, USA]

\section{Supplementary Materials}

645 Fig. S1 - Example gating strategy for sorting of IgG+ plasmablasts from human vaccinee

646 PBMCs.

647 Fig. S2 - Heavy chain CDR3 length after Vi-TT primary immunization or Vi-PS-boost

648 immunization

649 Fig. S3 - Sequence characteristics of BCRs from IgG-positive plasmablasts after Vi-TT primary

650 or Vi-PS boost immunization

651

652

653

654

655

656

657

658

659

Fig. S4 - Sequence characteristics of BCRs from IgG-positive plasmablasts after Vi-TT primary or Vi-PS boost immunization, focusing on Ig heavy chain variable regions

Fig. S5 - Monoclonal antibody binding to tetanus toxoid

Fig. S6 - Somatic hypermutations in sequences of Vi-positive monoclonal antibodies

Fig. S7 - Antibodies across different families have similar epitope specificities.

Fig. S8 - Full ${ }^{1} \mathrm{H}-{ }^{13} \mathrm{C}$ HSQC NMR analysis of Vi polysaccharide solutions as function of increasing $\mathrm{NH}_{4} \mathrm{OH}$ treatment.

Fig. S9 - Monoclonal antibodies derived by vaccination target non- $O$-acetylated Vi

Fig. S10 - Vi PS monoclonal antibodies recognize exposed and occluded epitopes 
Fig. S12 - Example of the ADCD gating strategy.

Fig. S13 - ADMP and ADCD potential are not associated with antibody on-rate.

Data S1. BCR sequencing information.

Data S2. Full kinetic and functional measurements for all monoclonal antibodies.

Data S3. Raw experimental data by figure panel for graphs with $\mathrm{n} \leq 25$ data points.

666

667

668

669

670

671

672

673

674

675

676

677

678

679

680

681

682

683

684

685

686

687

688

689

690

691

692

693

694

695

696

697

698

699

700

701

702

703

\section{References}

1. S. A. Plotkin, Correlates of protection induced by vaccination. Clin Vaccine Immunol 17, 1055-1065 (2010).

2. H. Kayhty, H. Peltola, V. Karanko, P. H. Makela, The protective level of serum antibodies to the capsular polysaccharide of Haemophilus influenzae type b. J Infect Dis 147, 1100 (1983).

3. C. E. Frasch, R. Borrow, J. Donnelly, Bactericidal antibody is the immunologic surrogate of protection against meningococcal disease. Vaccine 27 Suppl 2, B112-116 (2009).

4. S. Maciejewski, T. J. Ruckwardt, K. M. Morabito, B. M. Foreman, K. E. Burgomaster, D. N. Gordon, R. S. Pelc, C. R. DeMaso, S. Y. Ko, B. E. Fisher, E. S. Yang, D. Nair, K. E. Foulds, J. P. Todd, W. P. Kong, V. Roy, M. Aleshnick, S. D. Speer, N. Bourne, A. D. Barrett, M. C. Nason, M. Roederer, M. R. Gaudinski, G. L. Chen, K. A. Dowd, J. E. Ledgerwood, G. Alter, J. R. Mascola, B. S. Graham, T. C. Pierson, Distinct neutralizing antibody correlates of protection among related Zika virus vaccines identify a role for antibody quality. Sci Transl Med 12, (2020).

5. S. Pittala, K. Bagley, J. A. Schwartz, E. P. Brown, J. A. Weiner, I. J. Prado, W. Zhang, R. Xu, A. OtaSetlik, R. Pal, X. Shen, C. Beck, G. Ferrari, G. K. Lewis, C. C. LaBranche, D. C. Montefiori, G. D. Tomaras, G. Alter, M. Roederer, T. R. Fouts, M. E. Ackerman, C. Bailey-Kellogg, Antibody Fab-Fc properties outperform titer in predictive models of SIV vaccine-induced protection. Mol Syst Biol 15, e8747 (2019).

6. J. Feng, U. Gulati, X. Zhang, W. A. Keitel, D. M. Thompson, J. A. James, L. F. Thompson, G. M. Air, Antibody quantity versus quality after influenza vaccination. Vaccine 27, 6358-6362 (2009).

7. D. Kazmin, H. I. Nakaya, E. K. Lee, M. J. Johnson, R. van der Most, R. A. van den Berg, W. R. B allou, E. Jongert, U. Wille-Reece, C. Ockenhouse, A. Aderem, D. E. Zak, J. Sadoff, J. Hendriks, J. Wrammert, R. Ahmed, B. Pulendran, Systems analysis of protective immune responses to RTS,S malaria vaccination in humans. Proc Natl Acad Sci U S A 114, 2425-2430 (2017).

8. V. K. Mohan, V. Varanasi, A. Singh, M. F. Pasetti, M. M. Levine, R. Venkatesan, K. M. Ella, Safety and immunogenicity of a Vi polysaccharide-tetanus toxoid conjugate vaccine (Typbar-TCV) in healthy infants, children, and adults in typhoid endemic areas: a multicenter, 2-cohort, open-label, double-blind, randomized controlled phase 3 study. Clin Infect Dis 61, 393-402 (2015).

9. G. B. D. Typhoid, C. Paratyphoid, The global burden of typhoid and paratyphoid fevers: a systematic analysis for the Global Burden of Disease Study 2017. Lancet Infect Dis 19, 369-381 (2019).

10. V. K. Wong, S. Baker, D. J. Pickard, J. Parkhill, A. J. Page, N. A. Feasey, R. A. Kingsley, N. R. Thomson, J. A. Keane, F. X. Weill, D. J. Edwards, J. Hawkey, S. R. Harris, A. E. Mather, A. K. Cain, J. Hadfield, P. J. Hart, N. T. Thieu, E. J. Klemm, D. A. Glinos, R. F. Breiman, C. H. Watson, S. Kariuki, M. A. Gordon, R. S. Heyderman, C. Okoro, J. Jacobs, O. Lunguya, W. J. Edmunds, C. Msefula, J. A. Chabalgoity, M. Kama, K. Jenkins, S. Dutta, F. Marks, J. Campos, C. Thompson, S. Obaro, C. A. MacLennan, C. Dolecek, K. H. Keddy, A. M. Smith, C. M. Parry, A. Karkey, E. K. Mulholland, J. I. Campbell, S. Dongol, B. Basnyat, M. Dufour, D. Bandaranayake, T. T. Naseri, S. P. Singh, M. Hatta, P. Newton, R. S. Onsare, L. 
Isaia, D. Dance, V. Davong, G. Thwaites, L. Wijedoru, J. A. Crump, E. De Pinna, S. Nair, E. J. Nilles, D. P. Thanh, P. Turner, S. Soeng, M. Valcanis, J. Powling, K. Dimovski, G. Hogg, J. Farrar, K. E. Holt, G. Dougan, Phylogeographical analysis of the dominant multidrug-resistant H58 clade of Salmonella Typhi identifies inter- and intracontinental transmission events. Nat Genet 47, 632-639 (2015).

11. E. J. Klemm, S. Shakoor, A. J. Page, F. N. Qamar, K. Judge, D. K. Saeed, V. K. Wong, T. J. Dallman, S. Nair, S. Baker, G. Shaheen, S. Qureshi, M. T. Yousafzai, M. K. Saleem, Z. Hasan, G. Dougan, R. Hasan, Emergence of an Extensively Drug-Resistant Salmonella enterica Serovar Typhi Clone Harboring a Promiscuous Plasmid Encoding Resistance to Fluoroquinolones and Third-Generation Cephalosporins. MBio 9, (2018).

12. C. Jin, M. M. Gibani, M. Moore, H. B. Juel, E. Jones, J. Meiring, V. Harris, J. Gardner, A. Nebykova, S. A. Kerridge, J. Hill, H. Thomaides-Brears, C. J. Blohmke, L. M. Yu, B. Angus, A. J. Pollard, Efficacy and immunogenicity of a Vi-tetanus toxoid conjugate vaccine in the prevention of typhoid fever using a controlled human infection model of Salmonella Typhi: a randomised controlled, phase 2b trial. Lancet 390, 2472-2480 (2017).

13. M. Shakya, R. Colin-Jones, K. Theiss-Nyland, M. Voysey, D. Pant, N. Smith, X. Liu, S. Tonks, O. Mazur, Y. G. Farooq, J. Clarke, J. Hill, A. Adhikari, S. Dongol, A. Karkey, B. Bajracharya, S. Kelly, M. Gurung, S. Baker, K. M. Neuzil, S. Shrestha, B. Basnyat, A. J. Pollard, V. A. C. N. S. T. Ty, Phase 3 Efficacy Analysis of a Typhoid Conjugate Vaccine Trial in Nepal. N Engl J Med 381, 2209-2218 (2019).

14. K. P. Klugman, H. J. Koornhof, J. B. Robbins, N. N. Le Cam, Immunogenicity, efficacy and serological correlate of protection of Salmonella typhi Vi capsular polysaccharide vaccine three years after immunization. Vaccine 14, 435-438 (1996).

15. S. C. Szu, K. P. Klugman, S. Hunt, Re-examination of immune response and estimation of anti-Vi IgG protective threshold against typhoid fever-based on the efficacy trial of Vi conjugate in young children. Vaccine 32, 2359-2363 (2014).

16. L. C. Dahora, C. Jin, R. L. Spreng, F. Feely, R. Mathura, K. E. Seaton, L. Zhang, J. Hill, E. Jones, S. M. Alam, S. M. Dennison, A. J. Pollard, G. D. Tomaras, IgA and IgG1 Specific to Vi Polysaccharide of Salmonella Typhi Correlate With Protection Status in a Typhoid Fever Controlled Human Infection Model. Front Immunol 10, 2582 (2019).

17. C. Jin, J. Hill, B. M. Gunn, W. H. Yu, L. C. Dahora, E. Jones, M. Johnson, M. M. Gibani, R. L. Spreng, S. M. Alam, A. Nebykova, H. B. Juel, S. M. Dennison, K. E. Seaton, J. K. Fallon, G. D. Tomaras, G. Alter, A. J. Pollard, Vi-specific serological correlates of protection for typhoid fever. J Exp Med 218, (2021).

18. G. Dougan, S. Baker, Salmonella enterica serovar Typhi and the pathogenesis of typhoid fever. Annu Rev Microbiol 68, 317-336 (2014).

19. T. Wangdi, C. Y. Lee, A. M. Spees, C. Yu, D. D. Kingsbury, S. E. Winter, C. J. Hastey, R. P. Wilson, V. Heinrich, A. J. Baumler, The Vi capsular polysaccharide enables Salmonella enterica serovar typhi to evade microbe-guided neutrophil chemotaxis. PLoS Pathog 10, e1004306 (2014).

20. S. C. Szu, X. R. Li, A. L. Stone, J. B. Robbins, Relation between structure and immunologic properties of the Vi capsular polysaccharide. Infect Immun 59, 4555-4561 (1991).

21. F. Berti, R. De Ricco, R. Rappuoli, Role of O-Acetylation in the Immunogenicity of Bacterial Polysaccharide Vaccines. Molecules 23, (2018).

22. K. Hitri, M. M. Kuttel, G. De Benedetto, K. Lockyer, F. Gao, P. Hansal, T. R. Rudd, E. Beamish, S. Rijpkema, N. Ravenscroft, B. Bolgiano, O-acetylation of typhoid capsular polysaccharide confers polysaccharide rigidity and immunodominance by masking additional epitopes. Vaccine 37, 3866-3875 (2019).

23. Guidelines on the quality, safety and efficacy of typhoid conjugate vaccines. Who Tech Rep Ser 987, 101157 (2014).

24. B. Szewczyk, A. Taylor, Diversity of Vi-related antigens in the microcapsule of Salmonella typhi. Infect Immun 30, 661-667 (1980).

25. A. Qadri, S. Ghosh, G. P. Talwar, Monoclonal antibodies against two discrete determinants on Vi capsular polysaccharide. J Immunoassay 11, 235-250 (1990).

26. C. Henry, N. Y. Zheng, M. Huang, A. Cabanov, K. T. Rojas, K. Kaur, S. F. Andrews, A. E. Palm, Y. Q. Chen, Y. Li, K. Hoskova, H. A. Utset, M. C. Vieira, J. Wrammert, R. Ahmed, J. Holden-Wiltse, D. J. Topham, J. J. Treanor, H. C. Ertl, K. E. Schmader, S. Cobey, F. Krammer, S. E. Hensley, H. Greenberg, X. S. He, P. C. Wilson, Influenza Virus Vaccination Elicits Poorly Adapted B Cell Responses in Elderly Individuals. Cell Host Microbe 25, 357-366 e356 (2019). 
27. J. Zhou, K. R. Lottenbach, S. J. Barenkamp, D. C. Reason, Somatic hypermutation and diverse immunoglobulin gene usage in the human antibody response to the capsular polysaccharide of Streptococcus pneumoniae Type 6B. Infect Immun 72, 3505-3514 (2004).

28. Z. Chen, K. S. Cox, A. Tang, J. Roman, M. Fink, R. M. Kaufhold, L. Guan, A. Xie, M. A. Boddicker, D. McGuinness, X. Xiao, H. Li, J. M. Skinner, T. Verch, M. Retzlaff, K. A. Vora, Human monoclonal antibodies isolated from a primary pneumococcal conjugate Vaccinee demonstrates the expansion of an antigen-driven Hypermutated memory B cell response. BMC Infect Dis 18, 613 (2018).

29. S. A. Ehrhardt, M. Zehner, V. Krahling, H. Cohen-Dvashi, C. Kreer, N. Elad, H. Gruell, M. S. Ercanoglu, P. Schommers, L. Gieselmann, R. Eggeling, C. Dahlke, T. Wolf, N. Pfeifer, M. M. Addo, R. Diskin, S. Becker, F. Klein, Polyclonal and convergent antibody response to Ebola virus vaccine rVSV-ZEBOV. Nat Med 25, 1589-1600 (2019).

30. F. Gao, C. Swann, P. Rigsby, S. Rijpkema, K. Lockyer, A. Logan, B. Bolgiano, I. S. W. G. Vi, Evaluation of two WHO First International Standards for Vi polysaccharide from Citrobacter freundii and Salmonella enterica subspecies enterica serovar Typhi. Biologicals 57, 34-45 (2019).

31. A. Hjelholt, G. Christiansen, U. S. Sorensen, S. Birkelund, IgG subclass profiles in normal human sera of antibodies specific to five kinds of microbial antigens. Pathog Dis 67, 206-213 (2013).

32. D. J. Barrett, E. M. Ayoub, IgG2 subclass restriction of antibody to pneumococcal polysaccharides. Clin Exp Immunol 63, 127-134 (1986).

33. G. Vidarsson, G. Dekkers, T. Rispens, IgG subclasses and allotypes: from structure to effector functions. Front Immunol 5, 520 (2014).

34. B. Briney, A. Inderbitzin, C. Joyce, D. R. Burton, Commonality despite exceptional diversity in the baseline human antibody repertoire. Nature 566, 393-397 (2019).

35. L. Liu, A. H. Lucas, IGH V3-23*01 and its allele V3-23*03 differ in their capacity to form the canonical human antibody combining site specific for the capsular polysaccharide of Haemophilus influenzae type $b$. Immunogenetics 55, 336-338 (2003).

36. G. L. Zhang, M. M. Wei, C. C. Song, Y. F. Ma, X. J. Zheng, D. C. Xiong, X. S. Ye, Chemical synthesis and biological evaluation of penta- to octa-saccharide fragments of Vi polysaccharide from Salmonella typhi. Org Chem Front 5, 2179-2188 (2018).

37. S. C. Szu, X. R. Li, R. Schneerson, J. H. Vickers, D. Bryla, J. B. Robbins, Comparative immunogenicities of $\mathrm{Vi}$ polysaccharide-protein conjugates composed of cholera toxin or its B subunit as a carrier bound to high- or lower-molecular-weight Vi. Infect Immun 57, 3823-3827 (1989).

38. S. L. Harris, P. Fernsten, Thermodynamics and density of binding of a panel of antibodies to highmolecular-weight capsular polysaccharides. Clin Vaccine Immunol 16, 37-42 (2009).

39. M. Cygler, D. R. Rose, D. R. Bundle, Recognition of a cell-surface oligosaccharide of pathogenic Salmonella by an antibody Fab fragment. Science 253, 442-445 (1991).

40. G. T. Rijkers, E. A. Sanders, M. A. Breukels, B. J. Zegers, Infant B cell responses to polysaccharide determinants. Vaccine 16, 1396-1400 (1998).

41. A. Weintraub, Immunology of bacterial polysaccharide antigens. Carbohydr Res 338, 2539-2547 (2003).

42. C. A. Laferriere, R. K. Sood, J. M. de Muys, F. Michon, H. J. Jennings, Streptococcus pneumoniae type 14 polysaccharide-conjugate vaccines: length stabilization of opsonophagocytic conformational polysaccharide epitopes. Infect Immun 66, 2441-2446 (1998).

43. A. S. Abdelhameed, G. A. Morris, F. Almutairi, G. G. Adams, P. Duvivier, K. Conrath, S. E. Harding, Solution conformation and flexibility of capsular polysaccharides from Neisseria meningitidis and glycoconjugates with the tetanus toxoid protein. Sci Rep 6, 35588 (2016).

44. N. Macura, T. Zhang, A. Casadevall, Dependence of macrophage phagocytic efficacy on antibody concentration. Infect Immun 75, 1904-1915 (2007).

45. J. D. Steckbeck, I. Orlov, A. Chow, H. Grieser, K. Miller, J. Bruno, J. E. Robinson, R. C. Montelaro, K. S. Cole, Kinetic rates of antibody binding correlate with neutralization sensitivity of variant simian immunodeficiency virus strains. J Virol 79, 12311-12320 (2005).

46. J. T. Bates, C. J. Keefer, T. J. Utley, B. E. Correia, W. R. Schief, J. E. Crowe, Jr., Reversion of somatic mutations of the respiratory syncytial virus-specific human monoclonal antibody Fab19 reveal a direct relationship between association rate and neutralizing potency. J Immunol 190, 3732-3739 (2013).

47. J. Zhao, R. Nussinov, B. Ma, Antigen binding allosterically promotes Fc receptor recognition. MAbs 11, 58-74 (2019).

48. C. T. Su, W. H. Lua, W. L. Ling, S. K. Gan, Allosteric Effects between the Antibody Constant and Variable Regions: A Study of IgA Fc Mutations on Antigen Binding. Antibodies (Basel) 7, (2018). 
49. E. Jones, C. Jin, L. Stockdale, C. Dold, A. J. Pollard, J. Hill, A Salmonella Typhi Controlled Human Infection Study for Assessing Correlation between Bactericidal Antibodies and Protection against Infection Induced by Typhoid Vaccination. Microorganisms 9, (2021).

50. F. Qadri, F. Khanam, X. Liu, K. Theiss-Nyland, P. K. Biswas, A. I. Bhuiyan, F. Ahmmed, R. Colin-Jones, N. Smith, S. Tonks, M. Voysey, Y. F. Mujadidi, O. Mazur, N. H. Rajib, M. I. Hossen, S. U. Ahmed, A. Khan, N. Rahman, G. Babu, M. Greenland, S. Kelly, M. Ireen, K. Islam, P. O'Reilly, K. S. Scherrer, V. E. Pitzer, K. M. Neuzil, K. Zaman, A. J. Pollard, J. D. Clemens, Protection by vaccination of children against typhoid fever with a Vi-tetanus toxoid conjugate vaccine in urban Bangladesh: a cluster-randomised trial. Lancet 398, 675-684 (2021).

51. C. S. Waddington, T. C. Darton, C. Jones, K. Haworth, A. Peters, T. John, B. A. Thompson, S. A. Kerridge, R. A. Kingsley, L. Zhou, K. E. Holt, L. M. Yu, S. Lockhart, J. J. Farrar, M. B. Sztein, G. Dougan, B. Angus, M. M. Levine, A. J. Pollard, An outpatient, ambulant-design, controlled human infection model using escalating doses of Salmonella Typhi challenge delivered in sodium bicarbonate solution. Clin Infect Dis 58, 1230-1240 (2014).

52. Y. C. Tan, S. Kongpachith, L. K. Blum, C. H. Ju, L. J. Lahey, D. R. Lu, X. Cai, C. A. Wagner, T. M. Lindstrom, J. Sokolove, W. H. Robinson, Barcode-enabled sequencing of plasmablast antibody repertoires in rheumatoid arthritis. Arthritis Rheumatol 66, 2706-2715 (2014).

53. J. M. Volpe, L. G. Cowell, T. B. Kepler, SoDA: implementation of a 3D alignment algorithm for inference of antigen receptor recombinations. Bioinformatics 22, 438-444 (2006).

54. M. E. Ackerman, B. Moldt, R. T. Wyatt, A. S. Dugast, E. McAndrew, S. Tsoukas, S. Jost, C. T. Berger, G. Sciaranghella, Q. Liu, D. J. Irvine, D. R. Burton, G. Alter, A robust, high-throughput assay to determine the phagocytic activity of clinical antibody samples. J Immunol Methods 366, 8-19 (2011).

55. S. Fischinger, J. K. Fallon, A. R. Michell, T. Broge, T. J. Suscovich, H. Streeck, G. Alter, A highthroughput, bead-based, antigen-specific assay to assess the ability of antibodies to induce complement activation. J Immunol Methods 473, 112630 (2019).

56. W. R. Pearson, An introduction to sequence similarity ("homology") searching. Curr Protoc Bioinformatics Chapter 3, Unit3 1 (2013).

Acknowledgments: We offer our sincerest thanks to our volunteers for participating in the study; the Oxford Vaccine Group Typhoid Study Team, in particular Emma Plested for project managing the study, Svetlana Milca for her recruitment efforts, Liaquat Khan for assisting with the organization of the study; David Lalloo (chair) and members of the Data Safety and Monitoring Committee for providing study oversight and guidance; Bharat Biotech International Limited for supplying the investigational vaccine (TybarTCV); Professor Myron M Levine and the University of Maryland for provision of the original $S$. Typhi Quailes challenge strain; the Wellcome Trust for funding the development of the typhoid challenge model; The Division of Bacteriology from the National Institute of Biological Standards and Control (NIBSC) for provision of the 12/244 International Standard for Vi polysaccharide antigen and guidance on the de-Oacetylation process and NMR; members of the Center for Human Systems Immunology, including Dr. Sarah Mudrak for GH-VAP program management support, additional laboratory members Dr. Jonathan Li, Derrick Goodman, DeAnna Tenney, and Reshma Thomas for training and specimen management; and the Duke University NMR Spectroscopy Center for use of their instrumentation.

Funding: This study was supported by The Bill and Melinda Gates Foundation (OPP1084259 and OPP1188863 to Pollard), a grant for the Antibody Dynamics platform of the Global Health-Vaccine Accelerator Platforms (GH-VAP) from the Bill and Melinda Gates Foundation (OPP1151372 and OPP1210938 to Tomaras), and the NIHR Oxford 
92

93

95

Biomedical Research Centre. Additional support was provided by the Dean's Graduate Fellowship, Duke University.

Author contributions: LCD, MV, KW, CJ, GC, DK, YL, JH, AP, and GT designed the study. Laboratory experiments were carried out by LCD, MV, KW, and YL. Data was analyzed and interpreted by LCD, MV, KW, LS, GC, EG, DK, YL, JH, BGB, LDS, AP, and GT. LD and MV wrote the first draft. AP and GT edited, and all authors reviewed and approved the final version of the manuscript.

Competing interests: AJP is Chair of UK Dept. Health and Social Care's (DHSC) Joint Committee on Vaccination \& Immunization (JCVI), and is a member of the WHO's SAGE. AJP is an NIHR Senior Investigator. The views expressed in this article do not necessarily represent the views of DHSC, JCVI, NIHR, or WHO. He is chief investigator on clinical trials of Oxford University's COVID19 vaccine, funded by NIHR. Oxford University has entered a joint COVID19 vaccine development partnership with Astra Zeneca.

\section{Data and materials availability:}

Data included in this manuscript are included in the supplementary data. All data needed to evaluate the conclusions in the manuscript are present in manuscript or the Supplementary Materials.

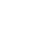

\section{7}

8

89

(n)

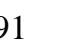

\section{4}



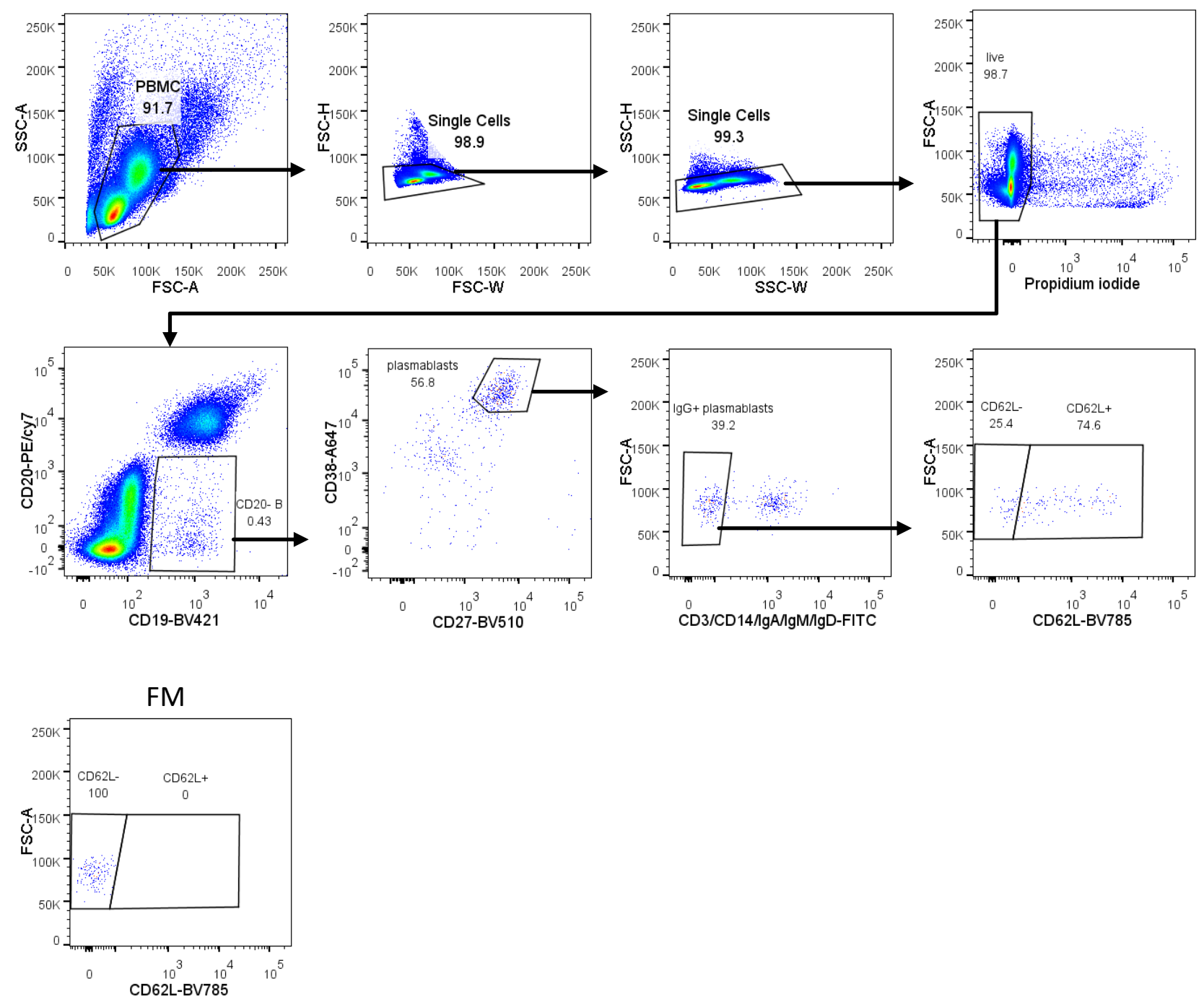

Fig. $\mathrm{S} 1$ - Example gating strategy for sorting of $\operatorname{lgG}^{+}$plasmablasts from human vaccinee PBMCs. 
935

936

937

938

939

940

941

942

943

944

945

946

947

948

949

950

951

952

953

954

955
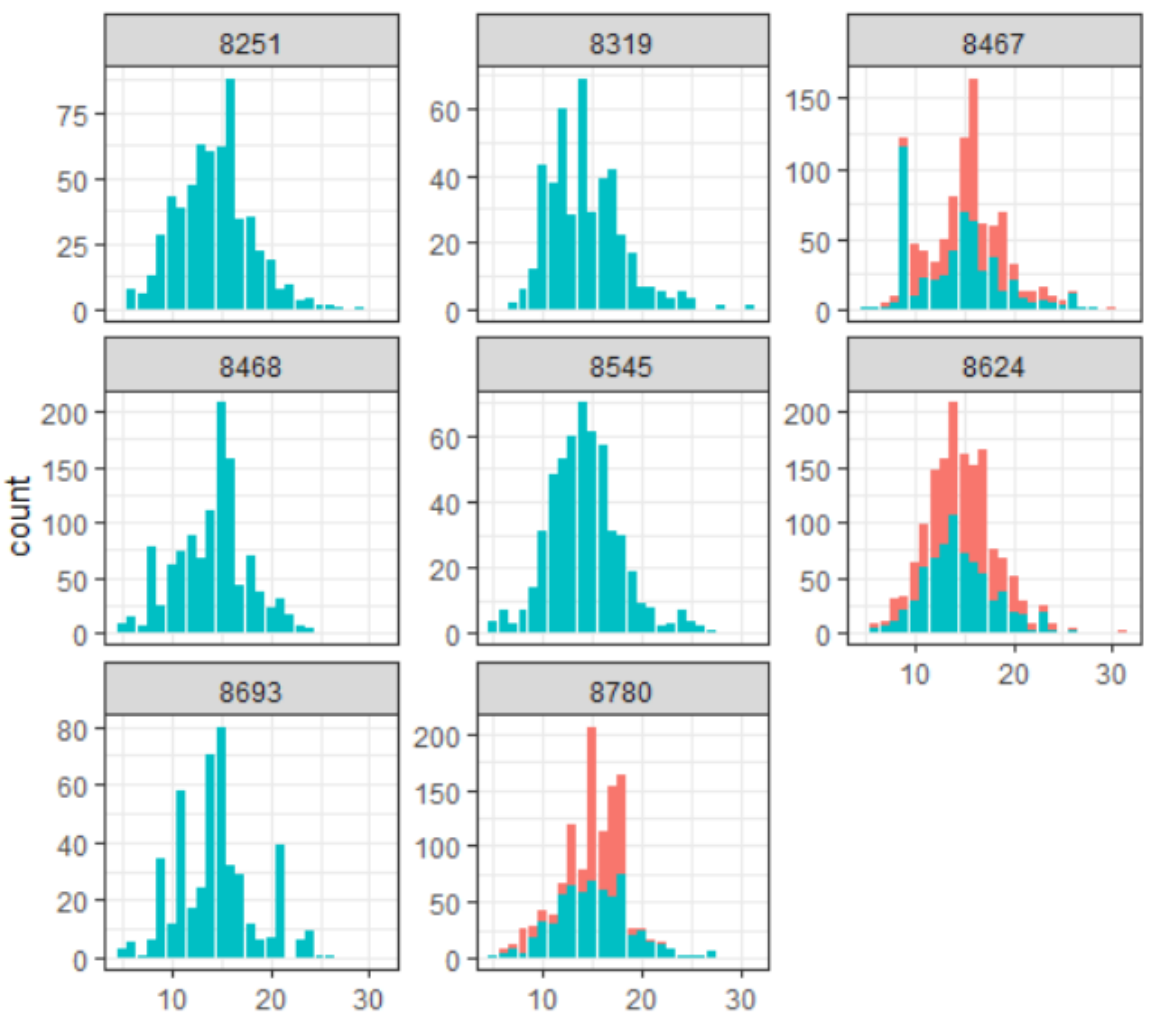

study_arm

Study Samples Post Prime

Study Samples Post Boost

Fig. S2 - Heavy chain CDR3 length after Vi-TT primary immunization or Vi-PS-boost immunization.
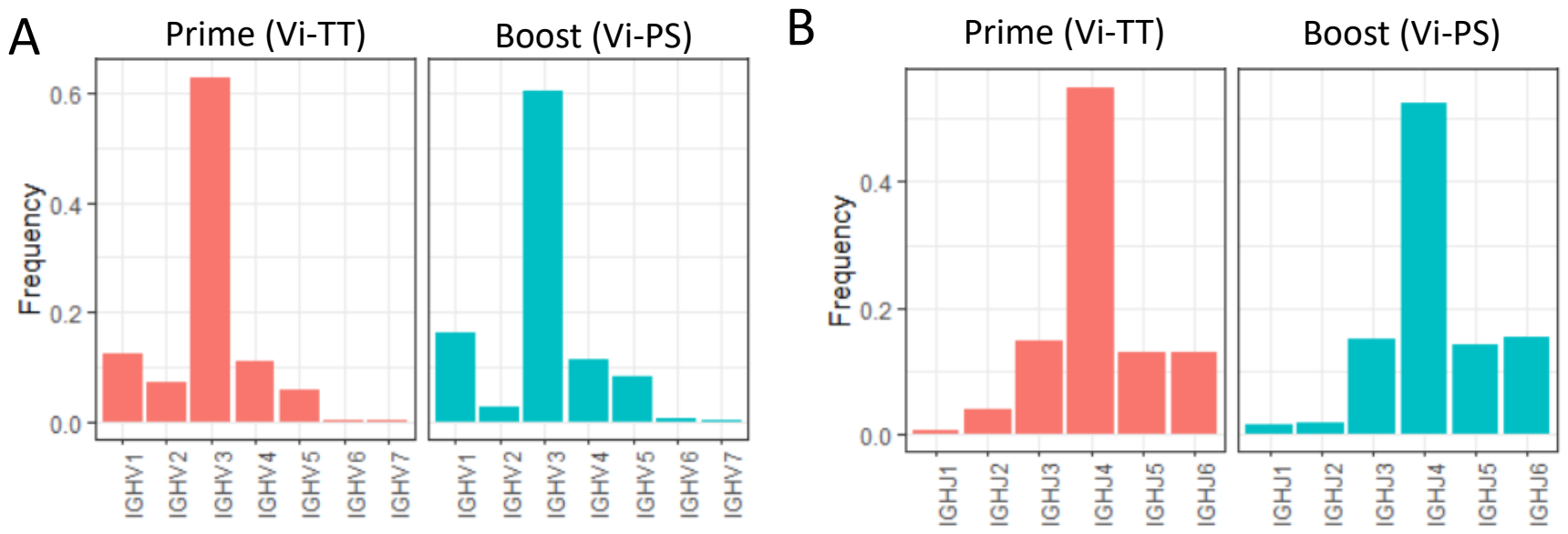

Fig. S3 - Sequence characteristics of BCRs from IgG-positive plasmablasts after Vi-TT primary or Vi-PS boost immunization A) Ig heavy chain variable region usage, showing samples that were paired between immunization $(n=3)$. B) Ig heavy chain junction region usage, showing samples that were paired between immunizations $(n=3)$. 
964

965

966

967

968

969

970

971

972

973

974

975

976

977

978

979

980

981

982

983

984

985

986

987

988

989

990

991

992

993

994

995
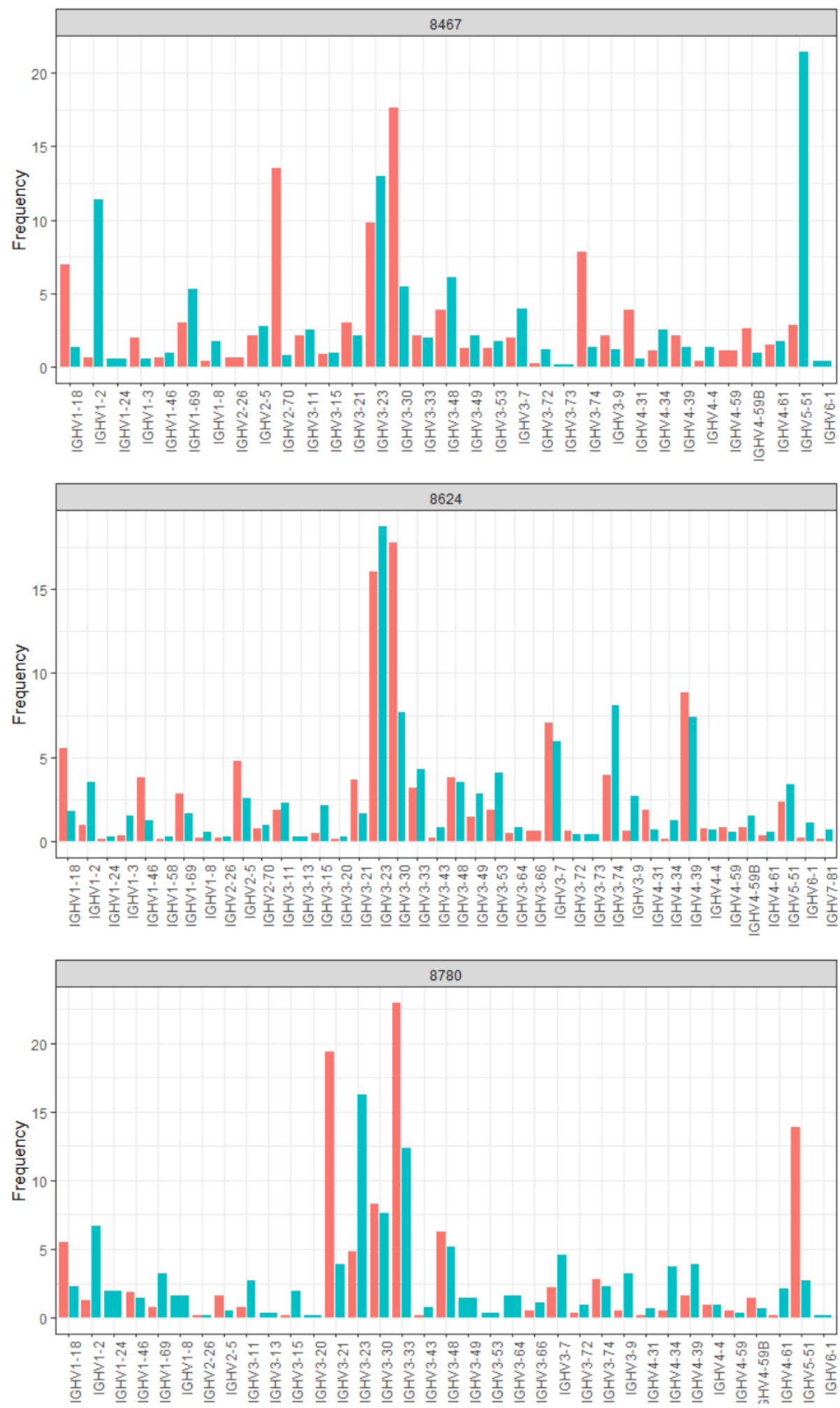

Prime (Vi-TT)

Boost (Vi-PS)

Fig. S4 - Sequence characteristics of BCRs from IgG-positive plasmablasts after Vi-TT primary or Vi-PS boost immunization, focusing Ig heavy chain variable regions Alg heavy chain variable region usage, showing the usage after the Vi-TT prime and Vi-PS boost immunizations for the 3 individuals for who data after both vaccines was available. 


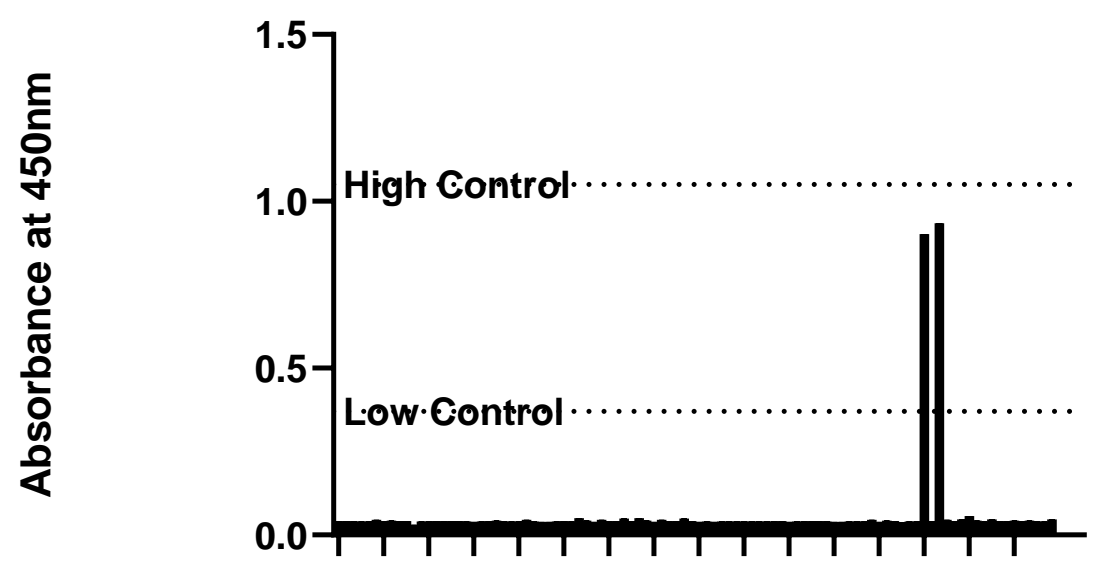

Monoclonal Antibody

996

997

998

999

1000

1001

1002

1003

1004

1005

1006

1007

1008

1009

1010

1011

1012

1013

1014

1015

1016

1017
A Heavy Chain

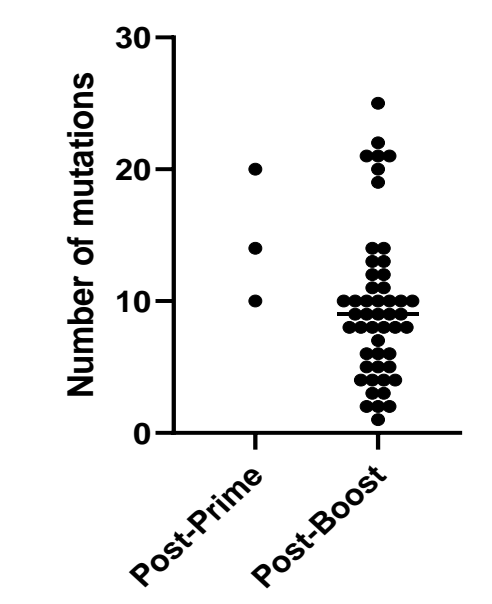

Fig. S5 - Monoclonal antibody binding to tetanus toxoid. Antibody binding was measured using the VaccZyme TetanusToxoid ELISA. The monoclonal antibodies were added to the plate at a concentration of $100 \mathrm{ng} / \mathrm{ml}$. Two monoclonal antibodies were shown to bind tetanus toxoid. The positive antibodies are $A B-007975$ and $A B-007990$. Both of these were negative for Vi antigen binding in the Vi polysaccharide ELISA.
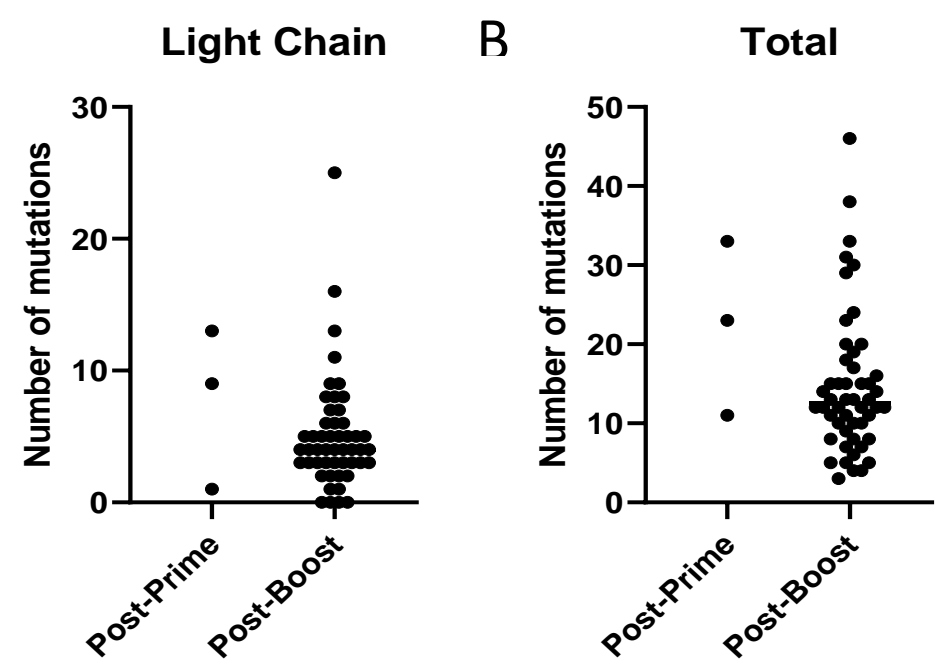

Fig. S6 - Somatic hypermutations in sequences of Vi-positive monoclonal antibodies. A) Number of somatic hypermutations in $\mathrm{V}$ heavy and $\mathrm{V}$ light chain after either primary immunization with Vi-TT $(n=3)$ or after a boost with Vi-PS $(n=50)$. B) Total number of somatic hypermutations in both chains after prime and boost. The number of mutations shown contains both silent and nonsilent mutations. The horizontal line indicates the median. 


\begin{tabular}{|c|c|c|c|c|c|c|c|}
\hline & & & & ${ }^{\text {nd }} n$ & $\mathrm{db}(\mathrm{A}$ & B-0C & \\
\hline & & & & ชิ & $\tilde{O}$ & J & \\
\hline & & & & $\underset{\mathfrak{N}}{\mathfrak{N}}$ & 怘 & 怘 & ঠ্口 \\
\hline & G1 & 7977 & & & & & \\
\hline & G2 & 7972 & & & & & \\
\hline E m & G3 & 7968 & & & & & \\
\hline & G4 & 7983 & & & & & \\
\hline & G5 & 7991 & & & & & \\
\hline
\end{tabular}

Fig. S7 - Antibodies across different families have similar epitope specificities. Crosscompetition between representative mAbs from each convergent family where ViPS antigen is immobilized at low density on biosensor, following by saturated binding of $1^{\text {st }} \mathrm{mAb}$, followed by binding of $2^{\text {nd }} \mathrm{mAb}$. Red indicates self-blocking, pink indicates cross-blocking, and green indicates no blocking.

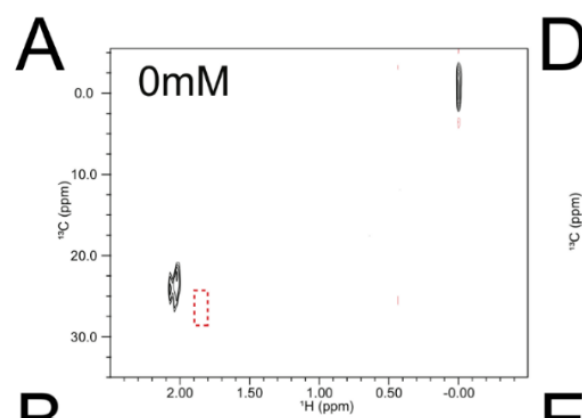

B

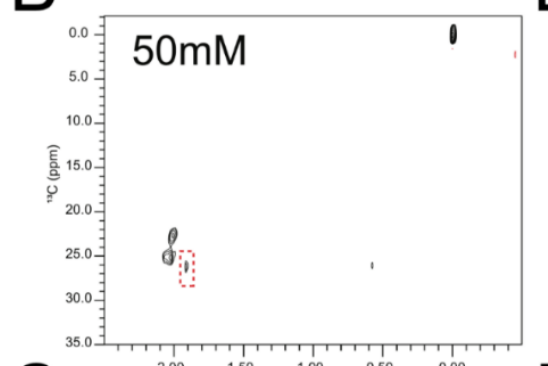

C

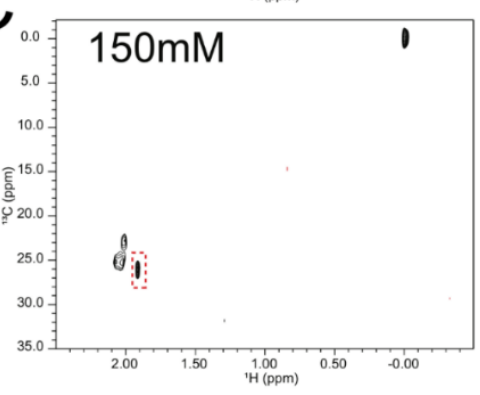

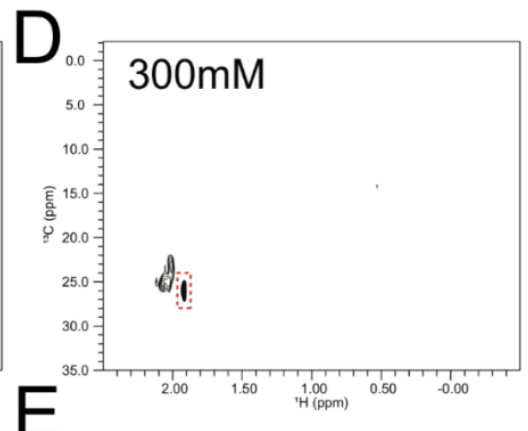
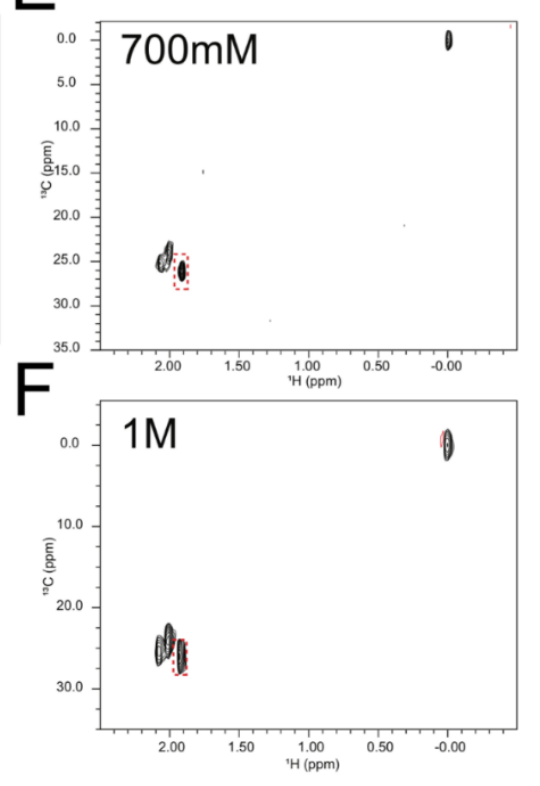
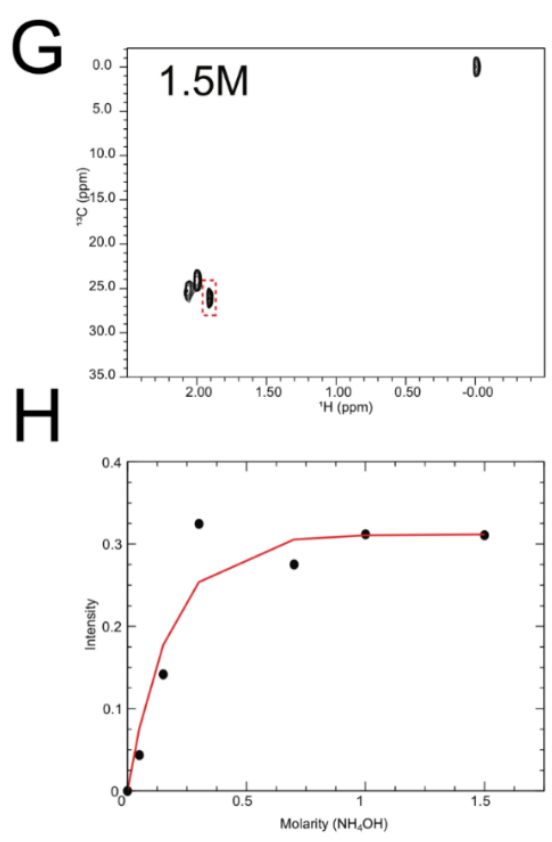

1027 
Fig. S8 - Full ${ }^{1} \mathrm{H}-{ }^{13} \mathrm{C}$ HSQC NMR analysis of Vi polysaccharide solutions as function of increasing $\mathrm{NH}_{4} \mathrm{OH}$ treatment. 2D NMR spectra of (A) untreated, (B) $0.05 \mathrm{M} \mathrm{NH}_{4} \mathrm{OH}$-treated, (C) $0.15 \mathrm{M} \mathrm{NH}_{4} \mathrm{OH}$-treated, (D) $0.3 \mathrm{M} \mathrm{NH}_{4} \mathrm{OH}$-treated, (E) $0.7 \mathrm{M} \mathrm{NH}_{4} \mathrm{OH}$-treated, (F) $1 \mathrm{M} \mathrm{NH}_{4} \mathrm{OH}$-treated, and (G) $1.5 \mathrm{M} \mathrm{NH}_{4} \mathrm{OH}$-treated $C$. freundii Vi solutions. The $\mathrm{x}$ - and $y$ - axis contain the NMR resonances arising from ${ }^{1} \mathrm{H}$ and ${ }^{13} \mathrm{C}$, respectively, representing ${ }^{1} \mathrm{H}-{ }^{13} \mathrm{C}$ direct bonds. Panel $\mathrm{H}$ represents the increasing signal (centered around $1.9 \mathrm{ppm}\left({ }^{1} \mathrm{H}\right)$ and $26.5 \mathrm{ppm}\left({ }^{13} \mathrm{C}\right)$ - dotted red box in each panel) as function of increasing amounts of $\mathrm{NH}_{4} \mathrm{OH}$ treatment. 
A

AB-007986-1 Binding ViPS

1074

1075

1076

1077

1078

1079

1080

1081

1082

1083

1084

1085

1086

1087

1088

1089

1090

1091

1092

1093

1094

1095

1096

1097

1098

1099

1100

1101

1102

1103

1104

1105

1106

1107

1108

1109

1110

1111

1112

1113

1114

1115

1116

1117

1118

B

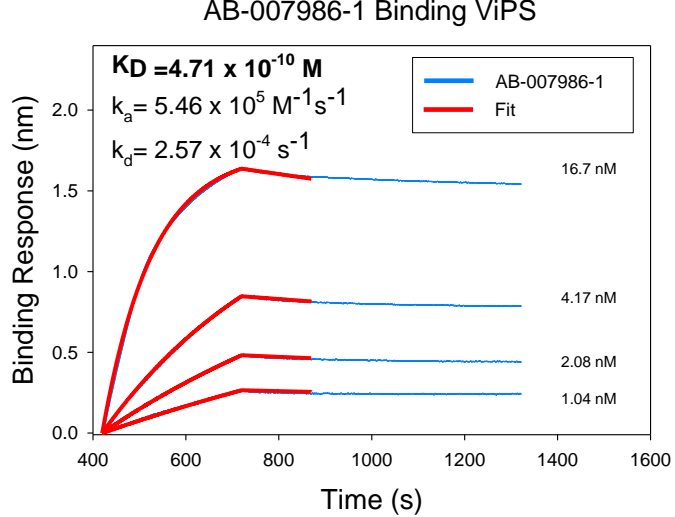

AB-007981-1 Binding ViPS

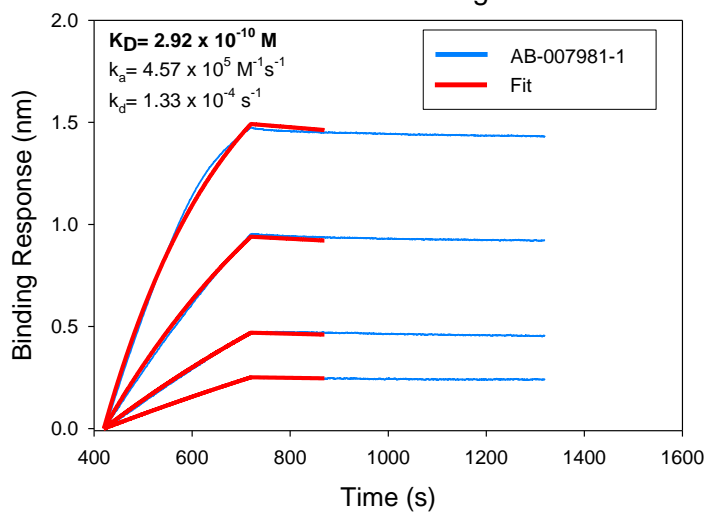

AB-007985-1 Binding ViPS

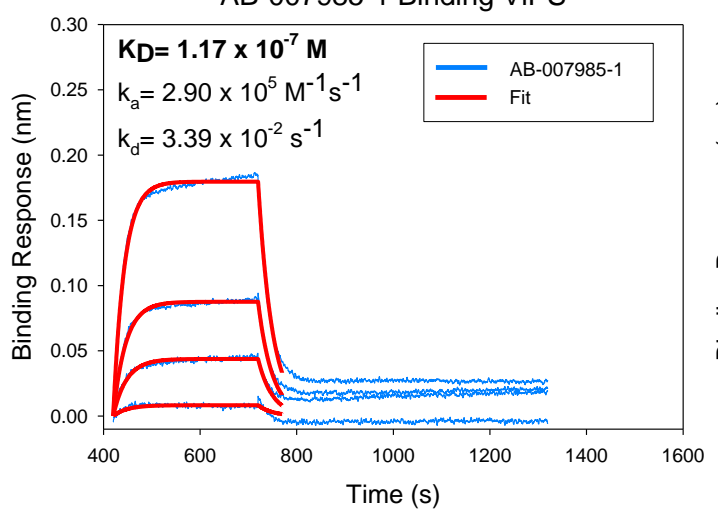

AB-007973-1 Binding ViPS

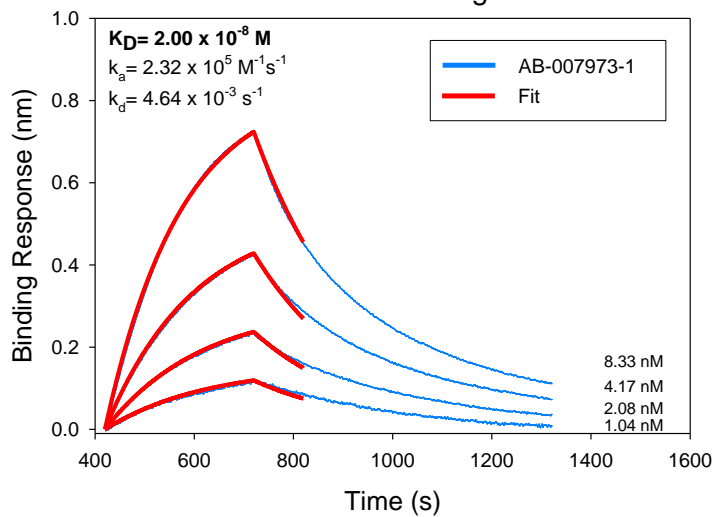

AB-007986-1 Binding De-O-ViPS

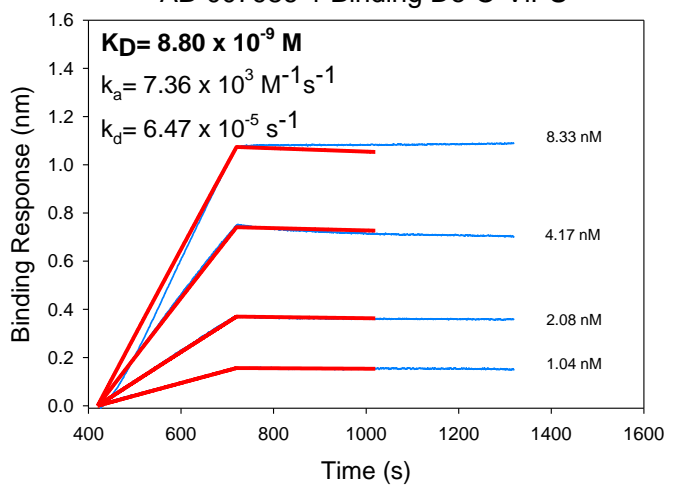

AB-007981-1 Binding De-O-ViPS

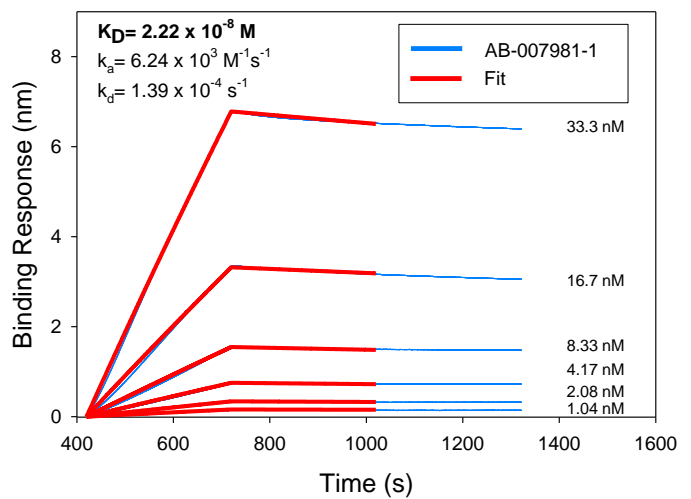

AB-007985-1 Binding De-O-ViPS

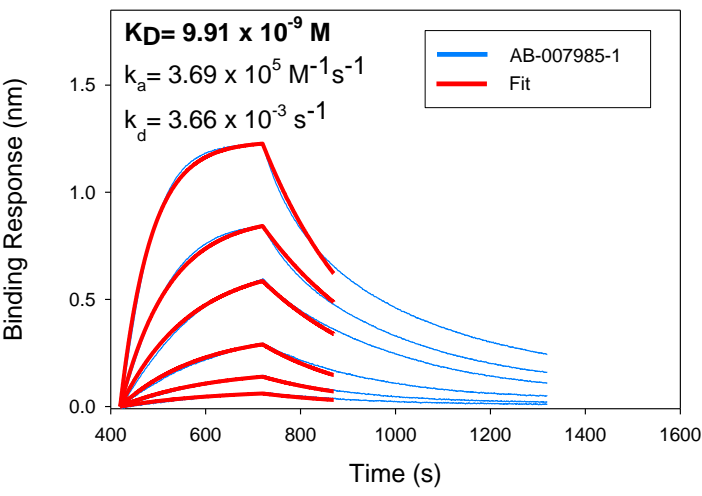

AB-007973-1 Binding De-O-ViPS

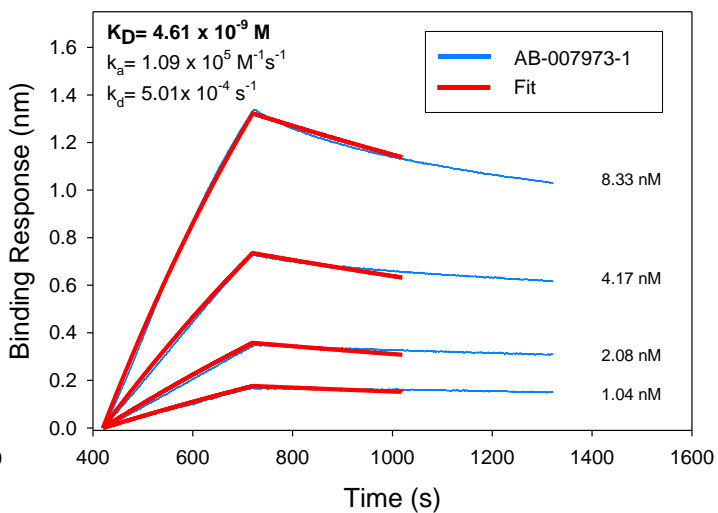

1119 
Fig. S9 - Monoclonal antibodies derived by vaccination target non-o-acetylated Vi. A) Binding sensorgrams of monoclonal antibodies that bind with similar avidity to Vi polysaccharide (left) and de-O-acetylated Vi polysaccharide (right) with corresponding avidity measurements indicated. B) Binding sensorgrams of monoclonal antibodies that bind with weaker avidity to Vi polysaccharide (left) compared to de-O-acetylated $\mathrm{Vi}$ polysaccharide (right) with corresponding avidity measurements indicated.

A
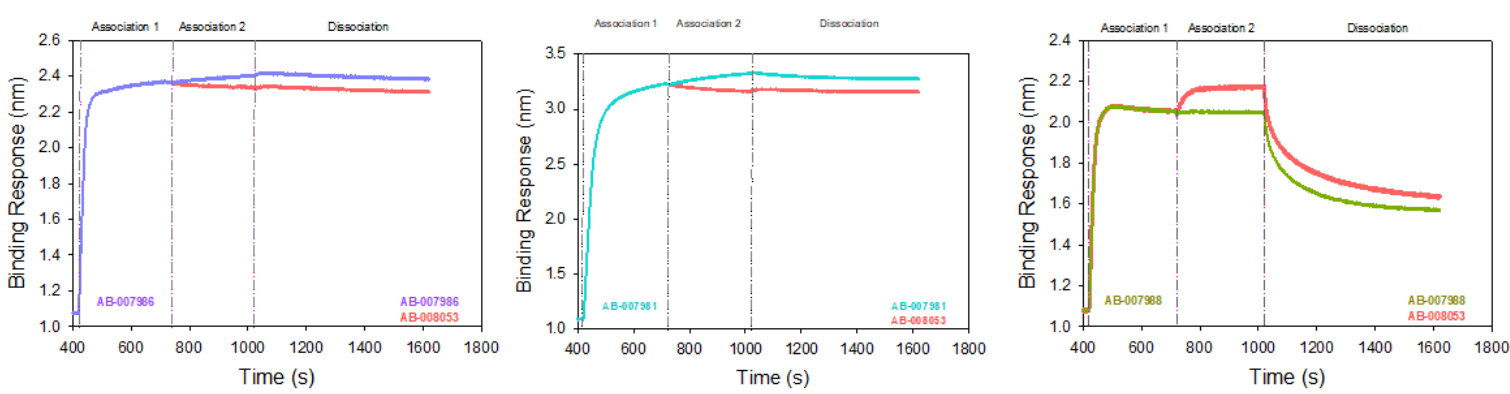

B
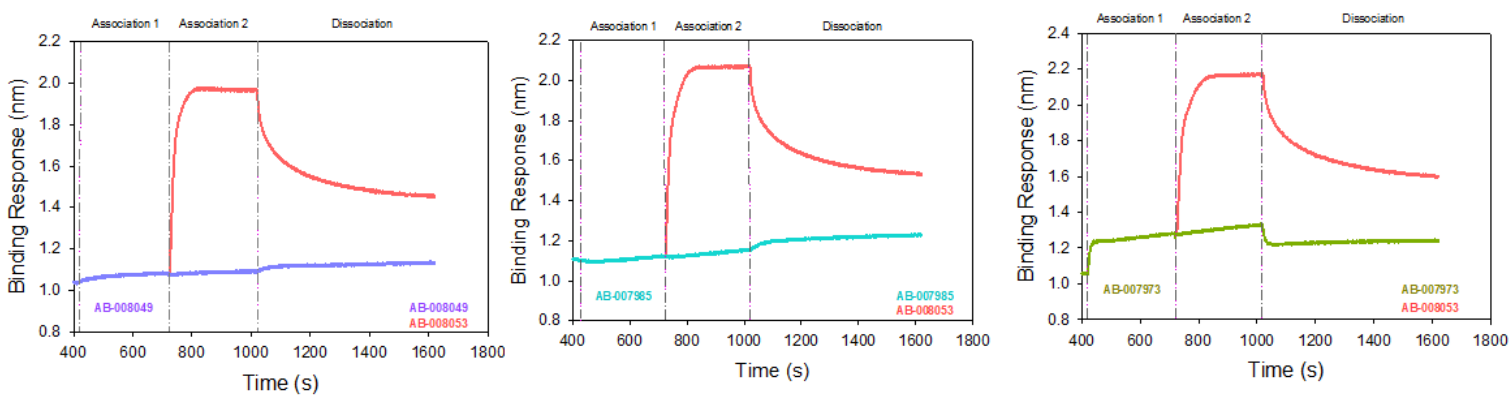

Fig. S10 - ViPS monoclonal antibodies recognize exposed and occluded epitopes Competition experiments performed by immobilizing low level ViPS antigen to biosensor, dipping into antibody 1 until binding is completely saturated, and then dipping into antiobdy 2. A) Competition of SEE mAbs in reverse orientation with SEE mAbs as antibody 1 and AB-008053 as antibody 2. B) Competition of SEO mAbs in reverse orientation with SEO mAbs as antibody 1 and AB-008053 as antibody 2. 

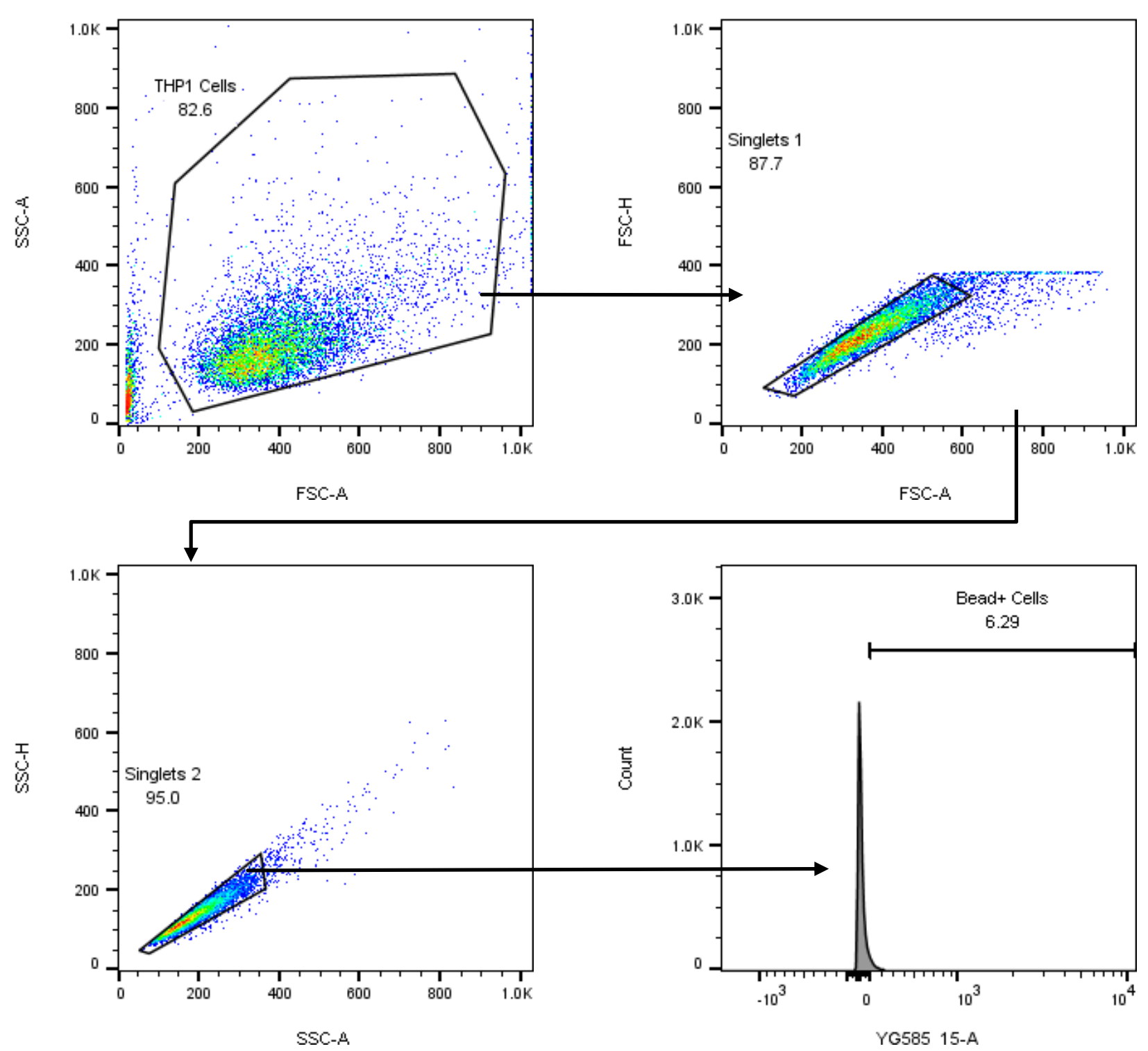

Fig. S11 - Example of the ADMP gating strategy. Initially, single cells are gated, followed by a selection of bead-positive cells. The gate for the bead-positive cells is based on a negative control THP-1 sample without any beads. Both the percentage of bead-positive cells and the intensity of the beads are used to calculate the phagocytic score. 

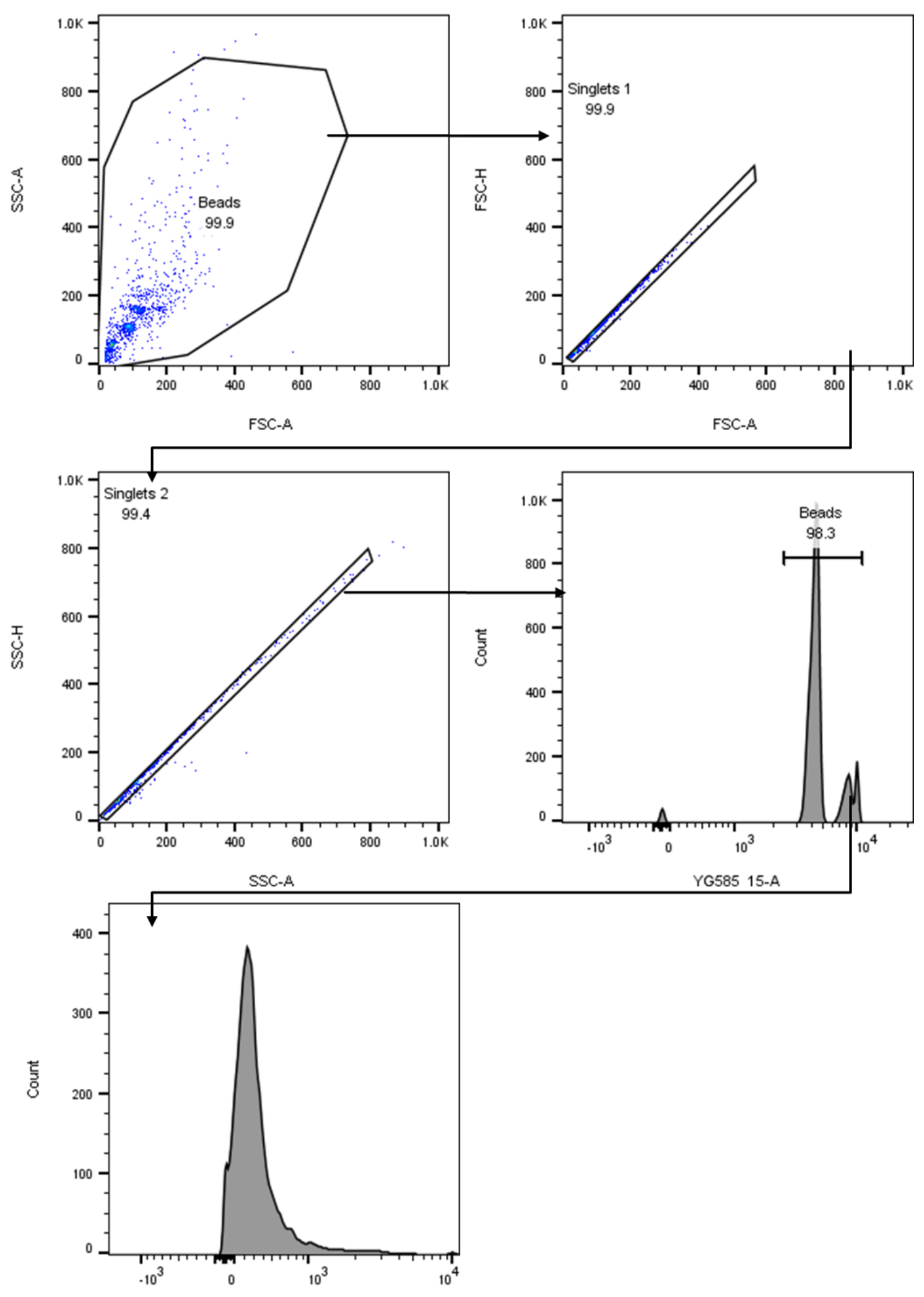

1157

B530 30-A 
Fig. S12 - Example of the ADCD gating strategy. Initially, single beads are gated, after which the anti-guinea pig C3-FITC intensity is measured using geometric mean fluorescent intensity.

A

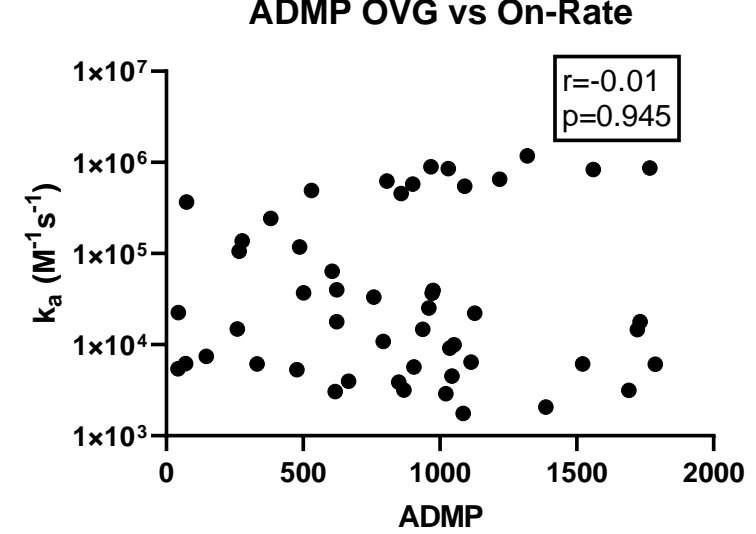

B

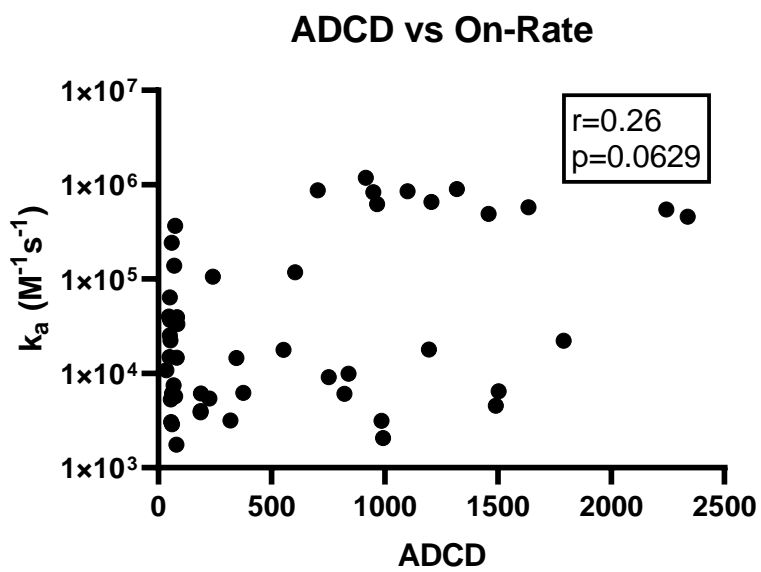

Fig. S13 - ADMP and ADCD potential are not associated with antibody on-rate. Spearman correlations of A) ADMP score and Vi on-rate. B) ADCD score and Vi on-rate. 